\title{
Inverse Scattering for Monochromatic Phaseless Measurements
}

\author{
Yuri Álvarez, Senior Member, IEEE, María García-Fernández, Student Member, IEEE, \\ Lorenzo Poli, Member, IEEE, Cebrián García-González, Paolo Rocca, Senior Member, IEEE, \\ Andrea Massa, Member, IEEE and Fernando Las-Heras, Senior Member, IEEE
}

\begin{abstract}
An inverse method and measurement setup for profile and constitutive parameters reconstruction from monochromatic phaseless information is presented. The method is based on the minimization of a cost function that relates the measured field with the one scattered by a model of the Object-UnderTest (OUT), where the position, contour, and constitutive parameters are the unknowns. As a result, phaseless information is directly related with the inverse problem unknowns, thus avoiding the need of an intermediate phase retrieval step. Due to the non-linear nature of the cost function, global optimization techniques such as the Particle Swarm Optimization (PSO) and Differential Evolution (DE) algorithms have been considered for cost function minimization. An exhaustive analysis of the cost function behavior as a function of the electric size of the OUT is presented, discussing the optimal OUT size where the proposed methodology provides accurate profile and constitutive parameters reconstruction. The proposed methodology is conceived to use it together with a simple, low-cost measurement setup for fast characterization of PEC and dielectric objects. Measurement examples are presented aiming to prove the feasibility of the described measurement setup.
\end{abstract}

Index Terms-Evolutionary algorithms, integral equations, inverse scattering, Method-of-Moments, optimization, phaseless measurements.

\section{INTRODUCTION}

Electromagnetic inverse scattering and imaging applications have been widely applied in different fields such as medical imaging [1],[2], security screening [3]-[5], through-the wallimaging [6], or non-destructive-testing [7],[8]. In all the cases, the idea is to take advantage of the scattered field information to extract the geometry and/or the constitutive parameters of the scenario-under-test. Recent advances in radiofrequency equipment and processing techniques have allowed the development of complex electromagnetic scanners, capable of providing real-time high resolution images. Examples can

Manuscript received July 5, 2016. This work has been supported by the Campus the Excelencia Internacional - Ayudas de Movilidad de Excelencia Banco Santander 2015 para Docentes e Investigadores de la Universidad de Oviedo en centros extranjeros; by the Ministerio de Economía y Competitividad - Gobierno de España under projects TEC 2014-54005-P (MIRIIEM) and MINECO-15-TEC2014-55290-JIN (PORTEMVISION); by the Gobierno del Principado de Asturias through the PCTI 2013-2017, FC-15-GRUPIN14-114.

Y. Álvarez, M. García-Fernández, C. García-Gonzalez, and F. Las-Heras are with the Area de Teoría de la Señal y Comunicaciones, Departamento de Ingeniería Eléctrica, Universidad de Oviedo, Campus Universitario de Gijón, 33203, Gijón (Asturias), Spain. (e-mail: \{yalopez, mariagarcia, cgarciag, flasheras\}@tsc.uniovi.es).

L. Poli, P. Rocca, and A. Massa are with the ELEDIA Research Center@DISI, University of Trento, Via Sommarive 5, Povo 38123 Trento - Italy (e-mail: lorenzo.poli@disi.unitn.it; paolo.rocca@unitn.it; andrea.massa@ing.unitn.it) be found in [1],[2] (tomographic system for breast cancer detection), [3]-[5] (millimeter-wave security screening), and [8] (tomographic system for inspection of dielectric materials).

The cited examples make use of the scattered field amplitude and phase information. However, phase measurement can be challenging at high frequency bands, requiring accurate and thus expensive radiofrequency hardware. Furthermore, phase measurement is quite sensitive to thermal drift.

Aiming to avoid direct measurement of the phase, several phaseless methods have been developed [9]-[15]: . On the one hand, holographic techniques [9] provide a fast, accurate retrieval of the phase taking advantage of the interference pattern created by a reference wave and the field scattered by the Object-Under-Test (OUT). On the other hand, iterative phase retrieval methods make use of the near field amplitude collected on two or more surfaces [12]. These are mainly based on a cost function minimization, so an adequate choice of the minimization strategy also influences the accuracy of the results. A detailed review of optimization techniques based on evolutionary algorithms has been presented in [16], concluding that Particle Swarm Optimization (PSO) and Differential Evolution (DE) are well-suited for nonlinear cost function minimization [17],[18].

Some of the aforementioned imaging systems are based on broadband measurements, where holographic techniques have been shown to be an effective solution over iterative phase retrieval methods for phaseless systems implementation [9]. However, in case of monochromatic measurements the latter can still be competitive [10]-[15] enabling the development simple, low cost measurement setup, as for example, the one described in Section 5 of [19] (a picture is shown in Fig. 5 of the referenced contribution). A summary of the phaseless techniques for imaging system is presented in Table I of [9].

A review of monochromatic (or single-frequency) techniques for geometry and constitutive parameters retrieval is presented in Table I. They can be classified according to the following criteria: i) Material (Perfect Electric Conductor (PEC) or dielectric), ii) Inversion method, and iii) OUT parametrization. The most common OUT parametrization methods are Fourier Series, splines, and pixel-based. The latter has the disadvantage of requiring a larger number of unknowns to parametrize the OUT, which is proportional to the OUT size. Fourier Series and splines can be used to parametrize slow-varying aspect angle OUTs with few parameters, regardless of the OUT size. Concerning optimization methods for monochromatic imaging problems, it can be observed that 
evolutionary algorithms have been considered in most of the previous works: Genetic Algorithms [20]-[23], Differential Evolution, [17], and Particle Swarm Optimization [17].

Several iterative scalar inversion methods using the multifrequency multistatic dataset of [24] are compared in [20]. The benchmarked methods are: i) Diffraction Tomography (DT); ii) Modified Gradient in Field; iii) Contrast Source Inversion; and iv) Real-Coded Genetic Algorithm (RGA). Only i) and iv) are used with single-frequency measurements. Most of the examples in Table I consider OUT sizes (i.e. maximum dimension of the OUT) ranging from $D_{\text {OUT }}=0.1 \lambda$ to $D_{\text {OUT }}=5 \lambda$. The upper bound is mainly due to the fact that electrically large objects create a fast spatial-varying scattered field. Then, a cost function relating the scattered field and the field scattered by the OUT model will exhibit several local minima and, in consequence, cost function minimization methods can get easily trapped in those local minima. As an alternative, scattered field backpropagation techniques are suitable for imaging electrically large objects since they have low computational complexity and they are not limited by the OUT geometry, as they rely on pixel (2D) or voxel (3D) investigation domain discretization. However, they are based on a simple propagation model (in general, an exponential term) that prevents an efficient use of the scattered field information if compared to cost function-based techniques.

\section{A. Aim and Scope}

Whereas holographic techniques have been widely applied for inverse scattering and imaging [9], iterative phase retrieval methods have been mainly restricted to antenna diagnostics and near field to far field transformation. The majority of phaseless techniques for profile and constitutive parameters reconstruction rely on a multi-stage strategy [11]-[15]: first, the phase of the scattered field is recovered, and then, an inverse scattering technique is applied. The main drawback of two-stage strategies is that the error on the retrieved phase may degrade the reconstruction accuracy of the second stage.

The novelties of this contribution are: i) Development and testing of a methodology for recovering the profile and constitutive parameters of PEC and homogeneous dielectric bodies from monochromatic scattered field amplitude-only measurements, avoiding the intermediate step of phase retrieval [11][15]. ii) Influence of the OUT electric size in the reconstruction technique accuracy, providing an estimate of the OUT size range that yields best reconstruction accuracy. iii) Proof of the feasibility of a simple, low-cost multistatic imaging system.

\section{Description of the Monochromatic Phaseless MEASUREMENT SETUP}

A simple monochromatic phaseless measurement setup for imaging applications can be implemented as depicted in Fig. 1. A bistatic setup is shown in Fig. 1 (a), where the transmitting and receiving antennas keep the same aspect angle with respect to the OUT. Fig. 1 (b) corresponds to a multistatic setup, in which the OUT is viewed under the same aspect angle by one of the two antennas (e.g. the receiving antenna). In the case of
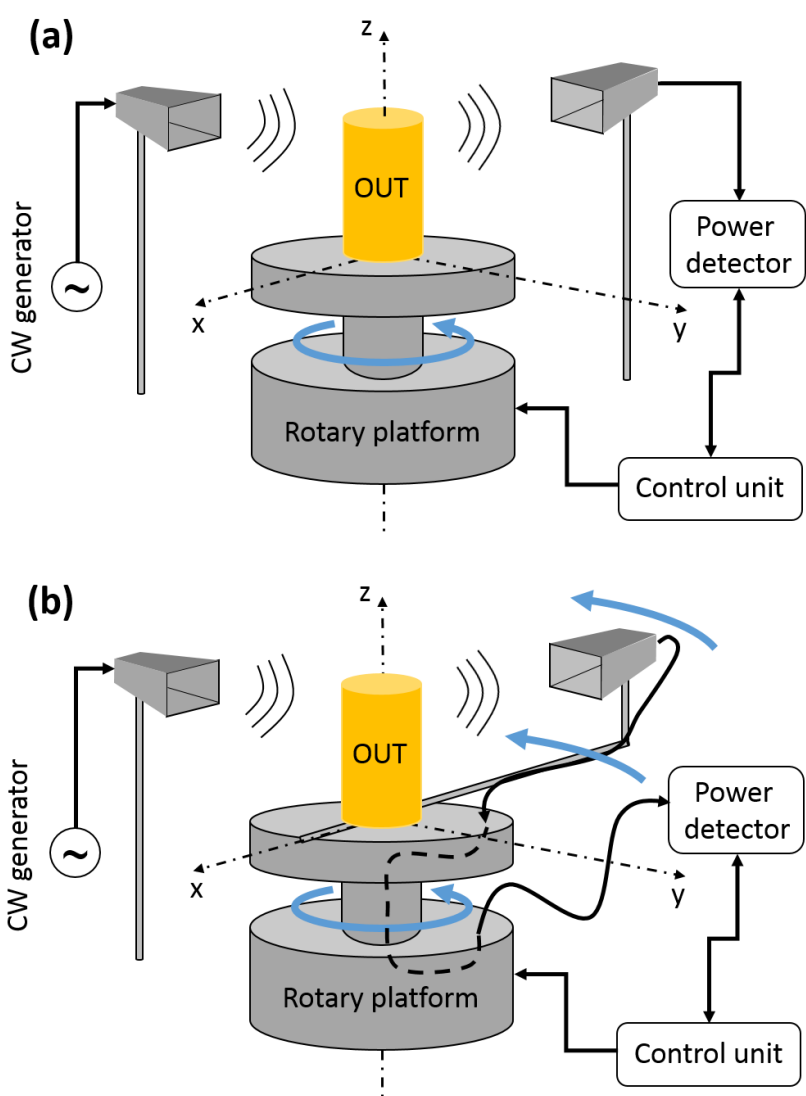

Fig. 1. Monochromatic multistatic phaseless measurement setup for electromagnetic imaging. (a) Bistatic. (b) Multistatic.

monostatic or bistatic setups, for each incident direction, only one scattered field sample is collected. However, for multistatic setups, the scattered field associated to an incident direction is collected in a set of points around the OUT, thus increasing the amount of available information about the OUT. In the case of phaseless monochromatic measurement setups, there is not multifrequency nor phase information, so a setup that maximizes the spatial or angular information, as the multistatic shown in 1 (b), is preferred over monostatic or bistatic setups.

Practical implementation of the measurement setup depicted in Fig. 1 (b) requires a continuous wave $(\mathrm{CW})$ generator, a transmitting antenna, a receiving antenna, a power detector, a rotary platform, and a control unit that synchronizes the measurement of the scattered field with the movement of the rotary platform. The low-cost X-band ( $\mathrm{f}=9.4 \mathrm{GHz}$ ) hardware for antenna measurement setup described in [25] and conceived for educational purposes has been reused, as shown in Fig. 2 (a). In the proposed setup, the receiving antenna is mounted onto the rotary platform, so that the distance between the receiving antenna and the center of the rotary platform is $R_{o b s}=22 \mathrm{~cm}$. The transmitting antenna is placed at $R_{i n c}=26 \mathrm{~cm}$. The rotary platform allows an angular sampling rate ranging from $\triangle \phi_{o b s}=0.5$ to $\Delta \phi_{o b s}=2$. Due to the fact that the transmitting and the receiving antennas 


\begin{tabular}{|c|c|c|c|c|c|}
\hline Ref. & Method & Comp. & OUT parametrization & Measurement layout & $\begin{array}{c}\text { Frequency / } \\
\text { electr. size }\left(D_{O U T}\right)\end{array}$ \\
\hline$[20]$ & Diffraction Tomography (DT) & PEC & Pixel-based & $\begin{array}{c}\text { Circular Rx array [24] } \\
\text { Tx. pos: 36, Rx. pos: } 49\end{array}$ & $8,16 \mathrm{GHz}$ \\
\hline$[21]$ & Binary Genetic Algorithm (BGA) & PEC & Fourier Series, 9 unknowns & $\begin{array}{c}\text { Circular Rx array } \\
\text { Tx. pos: } 4 \text {, Rx. pos: } 48\end{array}$ & $\begin{array}{c}300 \mathrm{MHz} \\
D_{O U T}<1 \lambda\end{array}$ \\
\hline$[20]$ & Real-coded Genetic Alg. (RGA) & PEC & Fourier Series & $\begin{array}{c}\text { Circular Rx array[24] } \\
\text { Tx. pos: } 36, \text { Rx. pos: } 49\end{array}$ & $8,16 \mathrm{GHz}$ \\
\hline$[17]$ & $\begin{array}{c}\text { Differential Evolution (DE) } \\
\text { Particle Swarm Optimization (PSO) }\end{array}$ & PEC & splines, 10 unknowns & $\begin{array}{c}\text { Circular Rx array } \\
\text { Tx. pos: 7, Rx. pos: } 32 \\
\end{array}$ & $\begin{array}{c}\text { OUT size: } \\
D_{\text {OUT }} \sim 1.5 \lambda\end{array}$ \\
\hline$[20]$ & Diffraction Tomography (DT) & Dielectric & Pixel-based & $\begin{array}{c}\text { Circular Rx array[24] } \\
\text { Tx. pos: } 36, \text { Rx. pos: } 49\end{array}$ & $8,16 \mathrm{GHz}$ \\
\hline$[22]$ & Real-coded Genetic Alg. (RGA) & Dielectric & Pixel-based, 16x16 & $\begin{array}{c}\text { Circular array } \\
\text { Tx. pos: 4, Rx. pos: } 40\end{array}$ & $\begin{array}{c}6 \mathrm{GHz} \\
D_{O U T}: 0.7 \lambda \text { to } 1 \lambda\end{array}$ \\
\hline [19] & Real-coded Genetic Alg. (RGA) & Dielectric & Pixel-based,9x9 & $\begin{array}{c}\text { Circular array } \\
\text { Tx. pos: } 4 \text {, Rx. pos: } 81\end{array}$ & $D_{O U T}: 0.66 \lambda$ \\
\hline$[20]$ & Real-coded Genetic Alg. (RGA) & Dielectric & Fourier Series & Tx. pos: 4 , Rx. pos: 40 & $8,16 \mathrm{GHz}$ \\
\hline$[23]$ & $\begin{array}{l}\text { Simulated Annealing } \\
\text { Genetic Algorithm }\end{array}$ & Dielectric & Pixel-based, 20x20 & $\begin{array}{c}\text { Circular array } \\
\text { Tx. pos: } 4 \text {, Rx. pos: } 32\end{array}$ & $\begin{array}{l}9,10,11 \mathrm{GHz} \\
D_{\text {OUT }} \sim 1.5 \lambda\end{array}$ \\
\hline
\end{tabular}

Fresnel Institute dataset [20]: OUT sizes range from $D_{\text {OUT }}=45 \mathrm{~mm}$ to $D_{O U T}=100 \mathrm{~mm}$, that is from $D_{\text {OUT }}=0.15 \lambda$ to $D_{\text {OUT }}=0.33 \lambda$ at $\mathrm{f}=1 \mathrm{GHz}$, and from $D_{O U T}=2.4 \lambda$ to $D_{O U T}=5.33 \lambda$ at $\mathrm{f}=16 \mathrm{GHz}$.

TABLE I

MONOCHROMATIC INVERSE SCATTERING. STATE-OF-THE-ART.

are located in the sample 2D plane, there will be an angular interval where the transmitting antenna will be shadowed by the receiving antenna. For this purpose, the scattered field will not be acquired in the entire circumference, but from $\phi_{\text {obs }} \subset\left(\left[50^{\circ}, 52^{\circ}, \ldots, 310^{\circ}\right]+\phi_{\text {inc }}\right)$. Horn antennas with $G=15$ $\mathrm{dB}\left(\cos ^{q}\right.$ radiation pattern, with approximately 36 degrees beamwidth), matched in the $8-12 \mathrm{GHz}$ frequency band are considered for transmission and reception [26]. For the given beamwidth of 36 degrees, it can be considered that an OUT with size $\left(D_{O U T}\right)$ smaller than $14 \mathrm{~cm}, D_{O U T}<14 \mathrm{~cm}$, will be uniformly illuminated. The power detector is a basic coaxial detector that has a quadratic behavior for small received voltages [27]. Due to the coupling between the transmitting and the receiving antennas, the power measured at the power detector will be proportional to the amplitude of the total field, which is the sum of the incident or background field (i.e. when the OUT is not present) and the field scattered by the OUT.

The OUT is placed on top of the rotary platform, as shown in 2 (a)-(c). The setup is conceived for TM-polarized 2D problems, so objects with translation symmetry along the vertical axis are considered (e.g. prisms, cylinders). Aiming to validate the feasibility of the setup to perform scattered field measurements, three OUTs, a $D_{\text {OUT }}=2.2 \mathrm{~cm}(0.7 \lambda)$ metallic cylinder, Fig. 2 (a), a wax candle with $D_{O U T}=$ $11.3 \mathrm{~cm}(3.5 \lambda)$, Fig. 2 (b), and a wood stick with $D_{O U T}$ $=4.7 \mathrm{~cm}(1.5 \lambda)$, Fig. 2 (c), have been considered for testing the setup. The measured total field has been compared with a simulation result based on a 2D full-wave integral equation formulation, [28],[29]. The implemented formulation is presented in Section II of [31] and summarized in Section III-A of this contribution.

The measured received power as a function of the observation angle for $\phi_{i n c}=0^{\circ}$ is depicted in Fig. 3 (a)(c) (solid red line) for the PEC and dielectric objects. The total field calculated using the integral equation formulation is also shown for comparison purposes (Fig. 3 (a)-(c), solid blue line), considering a dielectric wax candle with relative permittivity $\varepsilon_{r}=2.1$ and conductivity $\sigma=0.02 \mathrm{~S} / \mathrm{m}$, and a wood stick with $\varepsilon_{r}=2.8$ and $\sigma=0.3 \mathrm{~S} / \mathrm{m}$. Differences between simulations and measurements are mainly due to: i) the uncertainty about the true $\varepsilon_{r}$ and $\sigma$ values of the chosen samples, ii) total field is also affected by reflections in the objects around the measurement setup, iii) the error due to considering a 3D problem as $2 \mathrm{D}$, and iv) misalignment between the transmitting antenna, the receiving antenna, and the axis of the rotary platform. A laser level has been used for accurate positioning and alignment, ensuring a depointing error below $0.5^{\circ}$. Finally, it must be taken into account that the microwave devices and components are conceived for educational purposes, so their tolerances are not as good as professional equipment.

\section{Methodology}

Once the measurement setup has been described and verified, next step is the development of a methodology capable of recovering the profile and constitutive parameters of the OUT from the measured received power. A methodology based on a cost function minimization is proposed. 

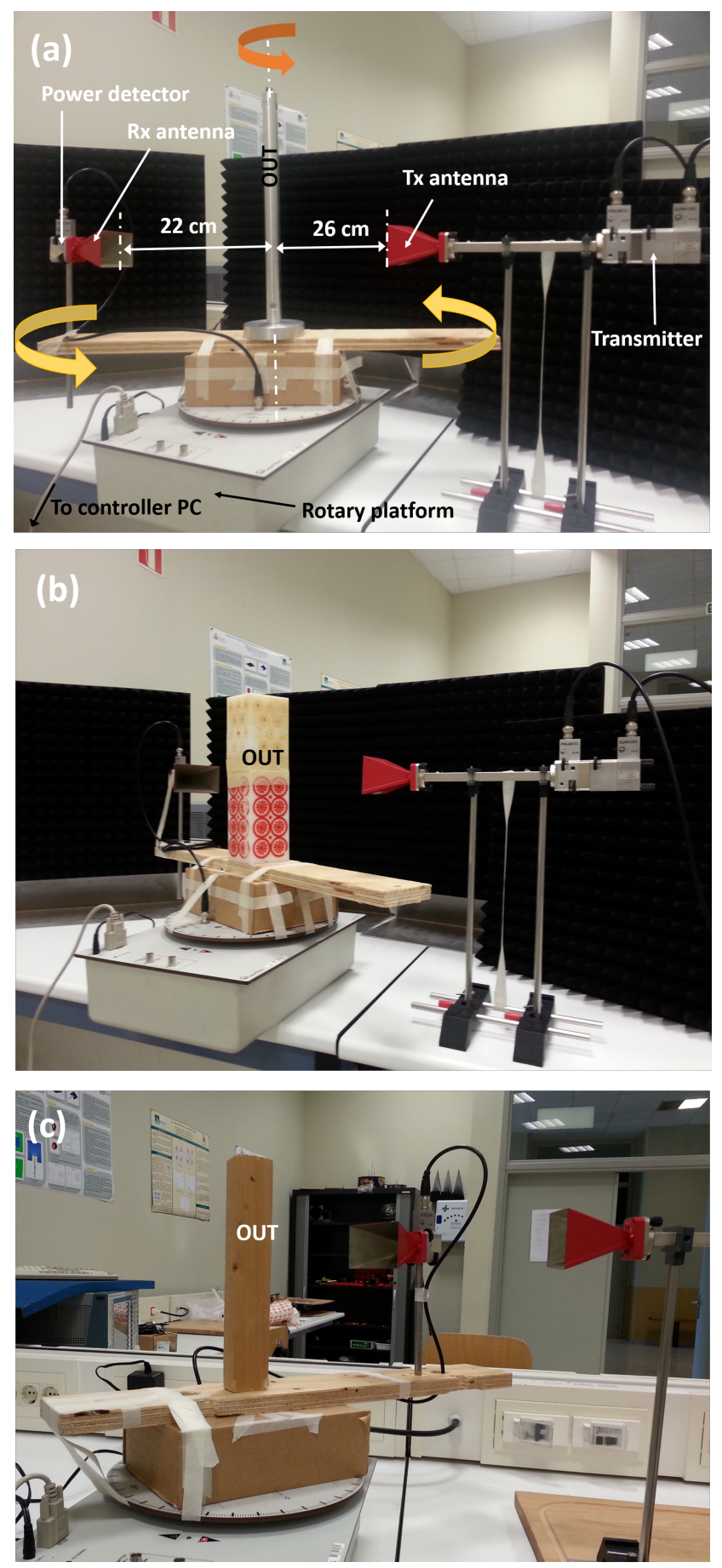

Fig. 2. Practical implementation of the multistatic phaseless measurement setup. OUT: metallic bar (a), wax candle (b), wood stick (c).
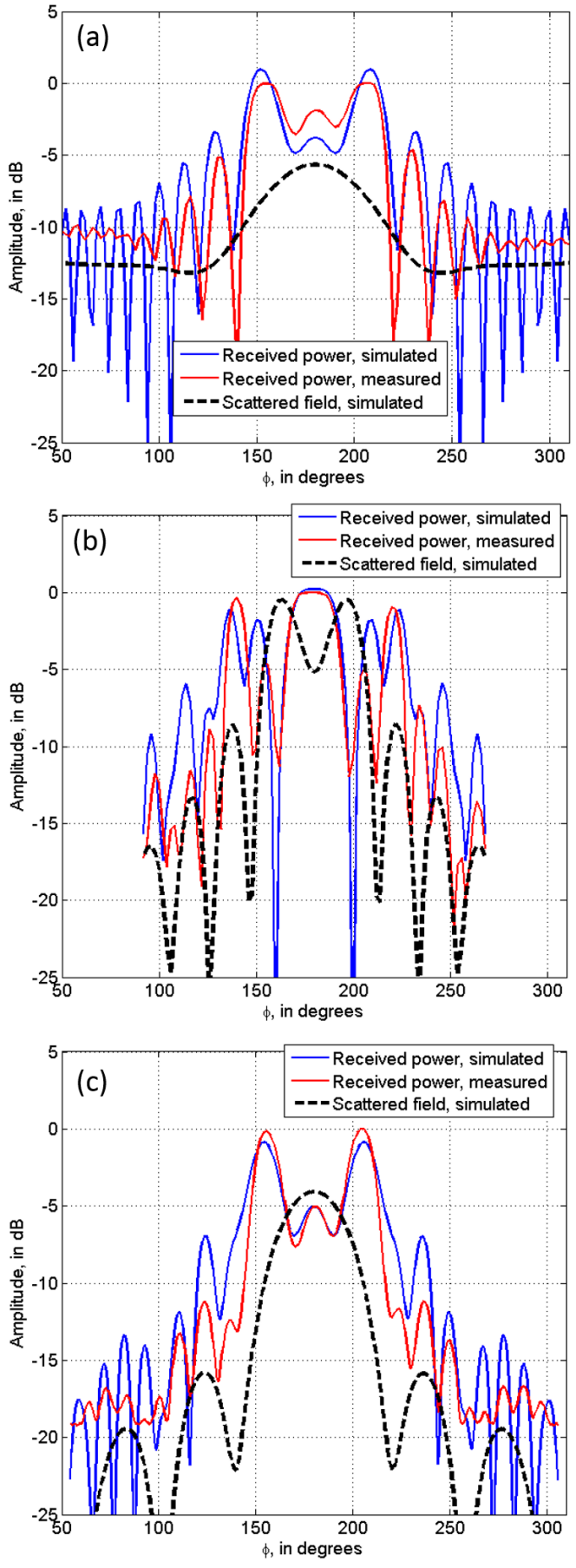

Fig. 3. Measured received power. (a) Metallic bar. (b) Wax candle. (c) Wood stick. 


\section{A. Formulation of the Problem}

For the sake of simplicity, a 2D problem is considered. This assumption can be valid for those bodies with constant crosssection along one dimension, as the one depicted in Fig. 2. In the case of amplitude and phase are available, a cost function $C F_{A P}(X)$ that relates the measured $S_{21}$ parameter for every $k$-th incidence and every $n$-th observation point, $S_{21, n, k}^{\text {scatt,meas }}$, to the simulated one, $S_{21, n, k}^{\text {scatt, sim }}$, can be established, similarly as proposed in [17], [21]:

$C F_{A P}(X)=\frac{\sum_{k=1}^{K} \sum_{n=1}^{N}\left(\left\|S_{21, n, k}^{\text {scatt,meas }}-S_{21, n, k}^{\text {scatt }, \text { sim }}(X)\right\|^{2}\right)}{\sum_{k=1}^{K} \sum_{n=1}^{N}\left(\left\|S_{21, n, k}^{\text {scatt }, \text { meas }}\right\|^{2}\right)}$

where the $S_{21, n, k}^{\text {scatt,sim }}(X)$ parameter is given by Eq. (2):

$$
S_{21, n, k}^{\text {scatt }, \text { sim }}(X)=G_{n}^{R x} E_{n, k}^{s c a t t, s i m}(X)
$$

and $G_{n}^{R x}$ is the receiving antenna pattern for the $n$-th observation point.

$X$ represents the set of unknowns, which are: i) the OUT profile, parametrized using Pchip splines; ii) the center of the OUT, $\left(x_{c e n}, y_{c e n}\right)$; and iii) in the case of dielectric bodies, conductivity $\sigma$, and permittivity $\varepsilon_{r}$ are also set as unknowns. In order to reduce the number of unknowns, each spline control point is defined in polar format, $\rho, \phi$. The angular position of the spline control points, $\vec{\phi}$, is fixed, and the angular interval $\left[\begin{array}{ll}0 & 360)^{\circ}\end{array}\right.$ is sampled uniformly (e.g. for 12 control points, $\left.\vec{\phi} \subseteq[0,30,60, \ldots, 330]^{\circ}\right)$. Then, the radius of the control points $\vec{\rho}$, are the unknowns. As a result, $X=\left[\vec{\rho}\left(x_{c e n}, y_{c e n}\right) \sigma \varepsilon_{r}\right]$.

In those measurement setups where the background field (i.e. incident field) is not characterized or measured, the $S_{21}$ parameter is not proportional to the scattered field but to the total field (Eq. (3)). Then, the incident field, $E^{i n c}$, has to be characterized using an electromagnetic model of the transmitting antenna.

$$
\begin{gathered}
S_{21, n, k}^{s c a t t}=G_{n}^{R x} E_{n, k}^{t o t} \\
E_{n, k}^{t o t}=E_{n, k}^{i n c}+E_{n, k}^{s c a t t}
\end{gathered}
$$

The simulated scattered field that depends on the aforementioned OUT parameters, $E_{n, k}^{s c a t t, s i m}(X)$, is calculated using a $2 \mathrm{D}$ full-wave integral equation formulation. TM polarization is considered [28],[29]. In particular, the formulation presented in [30] and [31] for homogeneous dielectric bodies has been implemented, Eq. (4):

$$
\begin{gathered}
E_{n, k}^{s c a t t, s i m}(X)=-j k_{0} \eta_{0} \int_{C} J_{z}(u) G_{0}\left(u, u_{o b s}\right) d C+ \\
\int_{C} M_{t}(u)\left[\hat{n_{u}} \cdot \nabla G_{0}\left(u, u_{o b s}\right)\right] d C
\end{gathered}
$$

where $k_{0}$ and $\eta_{0}$ are the free-space wavenumber and intrinsic impedance; $u$ denotes the OUT contour points, $u \epsilon C$, and are defined by the corresponding spline $u=f\left(\vec{\rho},\left(x_{c e n}, y_{c e n}\right)\right)$, with $\hat{n_{u}}$ the normal vector; $u_{o b s}$ are the observation points; $G_{0}$ is the 2D free-space Green's function; and $J_{z}$ and $M_{t}$ are the surface electric and magnetic currents, calculated by solving the integral equations (5) and (6), e.g. using a 2D Method-of-Moments (MoM):

$$
\begin{gathered}
E_{n, k}^{i n c}(u)=M_{t}(u)+j k_{0} \eta_{0} \int_{C} J_{z}\left(u^{\prime}\right) G_{0}\left(u, u^{\prime}\right) d C+ \\
-\int_{C} M_{t}\left(u^{\prime}\right)\left[\hat{n_{u^{\prime}}} \cdot \nabla G_{0}\left(u, u^{\prime}\right)\right] d C, u, u^{\prime} \epsilon C \\
0=M_{t}(u)-j k_{1} \eta_{1} \int_{C} J_{z}\left(u^{\prime}\right) G_{1}\left(u, u^{\prime}\right) d C+ \\
+\int_{C} M_{t}\left(u^{\prime}\right)\left[\hat{n_{u^{\prime}}} \cdot \nabla G_{1}\left(u, u^{\prime}\right)\right] d C, u, u^{\prime} \epsilon C
\end{gathered}
$$

where $k_{1}, \eta_{1}$, and $G_{1}$ are the wavenumber. intrinsic impedance, and 2D Green's function inside the homogeneous dielectric scatterer (conductivity $\sigma$, and relative permittivity $\left.\varepsilon_{r}\right)$.

In the case of PEC objects, the integral equations to be solved can be simplified (only electric currents are considered, Section II of [17]), thus reducing the calculation time.

For phaseless measurements, the cost function (1) is modified as follows:

$$
C F(X)=\frac{\sum_{k=1}^{K} \sum_{n=1}^{N}\left(\left\|\left|S_{21, n, k}^{\text {scatt,meas }}\right|-\left|S_{21, n, k}^{\text {scatt }, \text { sim }}(X)\right|\right\|^{2}\right)}{\sum_{k=1}^{K} \sum_{n=1}^{N}\left(\left\|\left|S_{21, n, k}^{\text {scatt }, \text { meas }}\right|\right\|^{2}\right)}
$$

Cost functions (1) and (7) relate all the available scattered field information at the same time. Despite this is the preferred implementation that can be found in the literature, multistage strategies have also been implemented aiming to speedup cost function minimization convergence [18]. Concerning cost function minimization, they have a non-linear relationship between the known data $S_{21, n, k}^{s c a t t, m e a s}$ and the unknowns $X$, which means that linear optimizations methods cannot be applied. Nevertheless, several non-linear optimization techniques could be applied. Their suitability will be justified from the analysis of the cost function behavior.

\section{B. Problem Parameters}

Several simulation and measurement examples have been selected for validating the proposed methodology and measurement setup. The specifications listed in this subsection and in Table II apply to all the evaluated examples in Sections IV, $\mathrm{V}$, and VI, unless otherwise specified.

- Incidence angles: for $K=4, \phi_{i n c}=0^{\circ}, 90^{\circ}, 180^{\circ}$, and $270^{\circ}$; and for $K=9, \phi_{i n c}=0^{\circ}, 40^{\circ}, 80^{\circ}, 120^{\circ}, 160^{\circ}, 200^{\circ}$, $240^{\circ}, 280^{\circ}$, and $320^{\circ}$; transmitter placed at $R_{\text {inc }}=76 \mathrm{~cm}$ (except for Section VI, $R_{\text {inc }}=22 \mathrm{~cm}$ ).

- Stochastic global optimization techniques are considered for cost function minimization. Thus, a population of initial solutions or initial candidates is needed. The number of candidates on each initial population is $P=70$ for Section IV, VI, and PECs in Section V; $P=50$ for dielectrics in Section $\mathrm{V}$; and $P=80$ for Section V-C. 
- Candidates are randomly created according to a uniform distribution whose bounds are defined in Table II.

\section{Analysis of the Cost Function Behavior}

This section is devoted to analyze the behavior of the cost function (7) for PEC and dielectric bodies. The OUT profile considered in this section is depicted in Fig. 7 (a), and it will be the same for all the simulation-based examples of this manuscript. OUT sizes $\left(D_{O U T}\right)$ of $0.5 \lambda, 1 \lambda$, and $2 \lambda$, corresponding to the frequencies of $\mathrm{f}=4 \mathrm{GHz}, 8 \mathrm{GHz}$, and $16 \mathrm{GHz}$, are considered.

The finite dimensionality of the solution space (given by the number of unknowns) prevents from an exhaustive analysis of the behavior of the cost function (7) in the entire search space. However, it is possible to obtain an estimate of the cost function behavior in a particular direction of that search space. Given the set of parameters modeling the true OUT (i.e. those which gives the cost function global minimum), $X_{\text {actual }}$, and a random candidate of the initial population, $X_{\text {rand }}$, the solution set in the direction $X_{t}$ is constructed as:

$$
X_{t}=X_{\text {actual }} t+X_{\text {rand }}(1-t)
$$

where $t$ ranges from $t=-0.75$ to $t=1.25$. The goal is to analyze if cost function (7) presents local minima in several random directions $X_{t}$. If the cost function exhibits several local minima in these directions, then, the global optimization method used to find the global minimum can be trapped in a local minimum. Thus, the higher the number of local minima, the higher the risk of global minimization technique stagnation.

First, cost function evaluation results for a PEC OUT are depicted in Fig. 4. It can be observed that there are no significant discrepancies from $1 \lambda$ to $2 \lambda$ (Fig. 4 (a) and (b)), although the cost function is slightly flatter for $2 \lambda$. For 0.5 $\lambda$, Fig. 4 (a), cost function values are around 10 times smaller than for larger OUT sizes. The justification can be obtained from the analysis of the fields scattered by the OUT, depicted in Fig. 6 (a). For $0.5 \lambda$, the amplitude of the scattered field has little variation (maximum - minimum ratio is about $5 \mathrm{~dB}$ ). In consequence, electrically small OUTs will scatter slow spatialvarying fields where the OUT shape will have little impact in the scattered field pattern. That means that reconstructed OUT profiles different from the true one can still return low cost function values.

In the case of the dielectric object with $\varepsilon_{r}=3$ and $\sigma=0.01$ $\mathrm{S} / \mathrm{m}$, there are differences between cost functions results for $0.5 \lambda, 1 \lambda$, and $2 \lambda$, as depicted in Fig. 5 . For $0.5 \lambda$, Fig. 5 (a), cost function exhibits several local minima, increasing the risk of stagnation when searching for the global minimum. It can be noticed the difference with respect to the PEC case of size $0.5 \lambda$ (Fig. 4 (a)), where the cost function has a monotonic decrease towards the global minimum. Concerning the differences between $1 \lambda$ and $2 \lambda$ (Fig. 5 (b) and (c) respectively), cost function response exhibits local minima for $2 \lambda$ whereas it has a monotonic decrease for $1 \lambda$. By looking at the scattered field amplitude, Fig. 6 (b), scattered field spatial variation for $2 \lambda$ is faster than for $0.5 \lambda$, which is in connection with the larger electric size of the OUT.

From the analysis presented in this subsection, it can be concluded that the scattered field spatial bandwidth (Fig. 6 (c)) is related with the cost function behavior, mainly by the number of local minima. If the OUT is electrically small, the scattered field amplitude has almost no variation and thus, there is not enough information to accurately estimate the OUT profile. Opposite to this, electrically large scatterers exhibit fast-varying scattered field amplitude, resulting in a cost function with multiple local minima that increases the chances of the global optimization method to get stuck in local minima.

Another parameter that might influence the cost function behavior is the number of incident directions, $K$. Cost function (7) has been evaluated for $K=4$ and $K=9$ incidences, concluding that increasing the number of incidences has little impact in the behavior of the cost function, regardless of the OUTs electric size.

Results presented in this section show that cost function (7) has local minima in the case of dielectric bodies, with a smoother profile for PECs. Taking the dielectric case as the most restrictive one, then, it can be concluded that global optimization algorithms must be applied to minimize cost function (7), trying not to converge prematurely to local minima. Taking into account the guidelines presented in [16], and that the set of unknowns $X$ has real data, PSO and DE are chosen as the most suitable techniques for cost function (7) minimization.

\section{Simulation-Based Results}

Next, the capability to recover the profile of PEC and dielectric objects from amplitude-only information is tested. The two considered evolutionary algorithms, PSO and DE, will be first benchmarked using simulation-based examples (Section V) and then with measurements (Section VI) to evaluate their performance, as done in former contributions [16],[17] for minimizing cost function (1).

A detailed explanation about the implementation and control parameters of the aforementioned evolutionary algorithms, as well as a comparison of their performance have been presented in [16]-[18],[32]. For a fair comparison, general purpose implementations of DE [16] and PSO [33] algorithms, not tuned for a specific problem, are considered.

In brief, PSO and DE start from an initial population of candidates, randomly created according to a uniform distribution whose bounds are defined in Table II. At each iteration, these optimization techniques modify the parameters of the candidates according to a set of search rules, yielding a new set of candidates. These rules take into account the cost function value of each candidate, pre-defined parameters (correction factor and inertial weight in PSO; and crossover probability and scaling factor in DE) as well as random factors. This 
Part 1 of 2:

\begin{tabular}{|c|c|c|c|c|c|c|}
\hline Section & Data & $\begin{array}{l}\text { Constitutive } \\
\text { parameters }\end{array}$ & $S_{21}$ & OUT size, $D_{\text {OUT }}$ & $\begin{array}{c}\text { Number of } \\
\text { unknowns, } N_{X}\end{array}$ & $\begin{array}{l}\text { Acquisition angular } \\
\text { interval, } \phi_{o b s}\end{array}$ \\
\hline IV & Sim. & PEC & Ampl.-only & $0.5,1,2 \lambda$ & $\begin{array}{l}12 \text { control points } \\
+\left(x_{\text {cen }}, y_{\text {cen }}\right)\end{array}$ & $\phi_{o b s} \in[0,4,8, \ldots, 356]^{\circ}$ \\
\hline V-A & Sim. & PEC & Complex & $2 \lambda$ & 12 control points & $\phi_{\text {obs }} \in[0,4,8, \ldots, 356]^{\circ}$ \\
\hline \multirow{2}{*}{$\mathrm{V}-\mathrm{C}$} & \multirow{2}{*}{ Sim. } & \multirow{2}{*}{$\begin{array}{c}\sigma=0.0 \mathrm{~S} / \mathrm{m} \\
\varepsilon_{r}=2.0\end{array}$} & Complex & \multirow{2}{*}{$0.66 \lambda$} & $\begin{array}{c}12 \text { c.p. }+\left(x_{c e n}, y_{c e n}\right) \\
+\left(\sigma, \varepsilon_{r}\right)\end{array}$ & \multirow{2}{*}{$\phi_{\text {obs }} \in[0,4.5,9, \ldots, 355.5]^{\circ}$} \\
\hline & & & Ampl.-only & & $\begin{array}{c}12 \text { c.p. }+\left(x_{c e n}, y_{c e n}\right) \\
+\left(\sigma, \varepsilon_{r}\right)\end{array}$ & \\
\hline \multirow{3}{*}{ VI-B } & \multirow{3}{*}{ Meas. } & PEC & \multirow{3}{*}{ Ampl.-only } & $0.7 \lambda$ & $\begin{array}{l}6 \text { control points } \\
+\left(x_{c e n}, y_{c e n}\right)\end{array}$ & \multirow{2}{*}{$\begin{array}{c}\phi_{\text {obs }} \in \\
\left(\left[50^{\circ}, 52^{\circ}, \ldots, 310^{\circ}\right]+\phi_{i n c}\right)\end{array}$} \\
\hline & & $\begin{array}{c}\sigma=0.30 \pm 0.03 \mathrm{~S} / \mathrm{m}[34] \\
\varepsilon_{r}=2.5 \pm 0.1\end{array}$ & & $1.5 \lambda$ & $\begin{array}{c}12 \text { c.p. }+\left(x_{c e n}, y_{c e n}\right) \\
+\left(\sigma, \varepsilon_{r}\right)\end{array}$ & \\
\hline & & $\begin{array}{c}\sigma \text { not specified. } \\
\varepsilon_{r}=2.3 \pm 0.2[35],[36]\end{array}$ & & $3.5 \lambda$ & $\begin{array}{c}12 \text { c.p. }+\left(x_{c e n}, y_{c e n}\right) \\
+\left(\sigma, \varepsilon_{r}\right)\end{array}$ & $\begin{array}{c}\phi_{\text {obs }} \in \\
\left(\left[90^{\circ}, 92^{\circ}, \ldots, 270^{\circ}\right]+\phi_{\text {inc }}\right)\end{array}$ \\
\hline
\end{tabular}

Sim.: simulation. Meas.: measurements. c.p.: control points.

Part 2 of 2:

\begin{tabular}{|c|c|c|c|c|c|c|}
\hline Section & Data & $\begin{array}{c}\text { Circular acquisition } \\
\qquad R_{o b s}\end{array}$ & $\begin{array}{c}\text { Incidences } \\
K\end{array}$ & $\begin{array}{l}\text { Control points bounds } \\
\text { (in } \mathrm{cm} \text { ) }\end{array}$ & $\begin{array}{l}\text { Center bounds } \\
\quad \text { (in } \mathrm{cm} \text { ) }\end{array}$ & $\begin{array}{c}\text { Constitutive } \\
\text { param. bounds } \\
\sigma \text { in } \mathrm{S} / \mathrm{m}\end{array}$ \\
\hline \multirow{2}{*}{ IV } & \multirow{2}{*}{ Sim. } & \multirow{2}{*}{$R_{o b s}=15 \mathrm{~cm}$} & \multirow{2}{*}{$K=4$} & \multirow{2}{*}{$\rho \in[0.0025 \ldots 0.05]$} & \multirow{2}{*}{$\begin{array}{l}\left(x_{c e n}, y_{c e n}\right) \in \\
{[-0.05 \ldots 0.05]}\end{array}$} & Not applicable, PEC \\
\hline & & & & & & $\begin{array}{c}\sigma \in[0.0001 \ldots 1] \\
\varepsilon_{r} \in[1.5 \ldots 6]\end{array}$ \\
\hline \multirow[t]{2}{*}{ V-A } & \multirow[t]{2}{*}{ Sim. } & $\begin{array}{c}\text { (Complex) } \\
R_{o b s, 1}=15 \mathrm{~cm}\end{array}$ & \multirow[t]{2}{*}{$K=4$} & \multirow{2}{*}{$\rho \in[0.0025 \ldots 0.05]$} & \multirow{2}{*}{$\begin{array}{l}\left(x_{c e n}, y_{c e n}\right) \in \\
{[-0.05 \ldots 0.05]}\end{array}$} & \multirow[t]{2}{*}{ Not applicable, PEC } \\
\hline & & $\begin{array}{c}\text { (Ampl. only) } \\
R_{o b s, 1}=15 \mathrm{~cm} \\
R_{o b s, 2}=30 \mathrm{~cm}\end{array}$ & & & & \\
\hline V-B & Sim. & $\begin{array}{l}R_{o b s, 1}=15 \mathrm{~cm} \\
R_{o b s, 2}=30 \mathrm{~cm}\end{array}$ & $K=4$ & $\rho \in[0.0025 \ldots 0.05]$ & $\begin{array}{c}\left(x_{c e n}, y_{c e n}\right) \in \\
{[-0.05 \ldots 0.05]}\end{array}$ & $\begin{array}{c}\sigma \in[0.0001 \ldots 1] \\
\varepsilon_{r} \in[1.5 \ldots 6] \\
\end{array}$ \\
\hline V-C & Sim. & $R_{o b s}=0.83 \lambda$ & $K=4$ & $\rho \in[0.0 \ldots 0.5] \lambda$ & $\begin{array}{l}\left(x_{c e n}, y_{c e n}\right) \in \\
{[-0.25 \ldots 0.25] \lambda}\end{array}$ & $\begin{array}{l}\sigma \in[0.0 \ldots 0.01] \\
\varepsilon_{r} \in[1.1 \ldots 3]\end{array}$ \\
\hline VI-A & Meas. & $R_{o b s}=72 \mathrm{~cm}$ & $K=9$ & $\rho \in[0.005 \ldots 0.03]$ & $\begin{array}{c}\left(x_{c e n}, y_{c e n}\right) \in \\
{[-0.04 \ldots 0.04]}\end{array}$ & $\begin{array}{l}\sigma \in[0.0001 \ldots 1] \\
\varepsilon_{r} \in[1.5 \ldots 6]\end{array}$ \\
\hline \multirow[t]{2}{*}{ VI-B } & \multirow[t]{2}{*}{ Meas. } & \multirow[t]{2}{*}{$R_{o b s}=22 \mathrm{~cm}$} & \multirow[t]{2}{*}{$K=4$} & $\begin{array}{c}\text { If } D_{O U T}<2 \lambda \\
\rho \in[0.005 \ldots 0.05]\end{array}$ & $\begin{array}{c}\text { If } D_{O U T}<2 \lambda \\
\left(x_{\text {cen }}, y_{\text {cen }}\right) \in \\
{[-0.04 \ldots 0.04]}\end{array}$ & \multirow[t]{2}{*}{$\begin{array}{l}\sigma \in[0.0001 \ldots 1] \\
\varepsilon_{r} \in[1.5 \ldots 6]\end{array}$} \\
\hline & & & & $\begin{array}{l}\text { If } D_{O U T}>2 \lambda \\
\rho \in[0.01 \ldots 0.07]\end{array}$ & $\begin{array}{c}\text { If } D_{O U T}>2 \lambda \\
\left(x_{\text {cen }}, y_{\text {cen }}\right) \in \\
{[-0.02 \ldots 0.02]}\end{array}$ & \\
\hline
\end{tabular}

TABLE II

PROBLEM-UNDER-TEST PARAMETERS. 


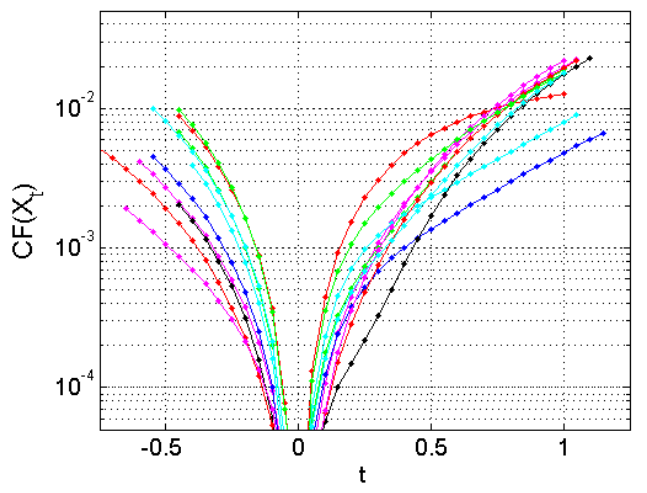

(a) CF (7) evaluation, OUT size, $D_{O U T}=0.5 \lambda$.

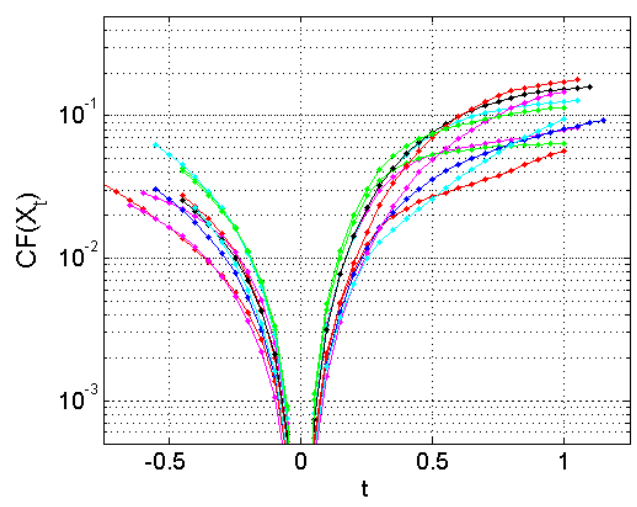

(b) CF (7) evaluation, OUT size, $D_{O U T}=1 \lambda$ ).



(c) CF (7) evaluation, OUT size, $D_{O U T}=2 \lambda$.

Fig. 4. Evaluation of cost function (7) for a PEC body. Results for 10 random candidates are shown.

procedure is repeated until a stopping condition (maximum number of iterations, algorithm stagnation, cost function below a certain threshold) is reached.

As a reminder, the simulations and measurements-based examples parameters have been described in Table II. In addition to this, the following parameters apply to all the evaluated examples of both Section V and Section VI:

- PSO and DE are stochastic global optimization methods. Thus, multiple initial population sets $(Q=100$ for PEC, and $Q=70$ for dielectrics) are considered, aiming to analyze the probability of convergence to the global

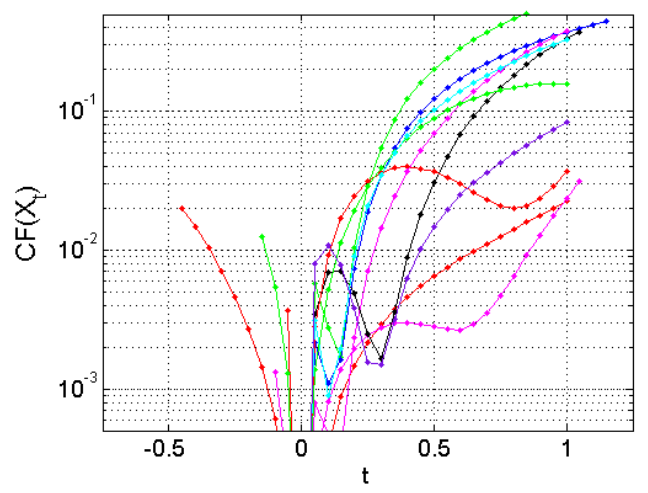

(a) CF (7) evaluation, OUT size, $D_{O U T}=0.5 \lambda$.

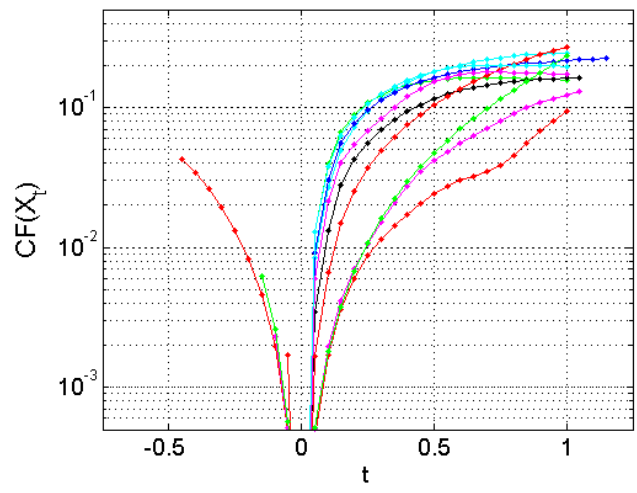

(b) CF (7) evaluation, OUT size, $D_{O U T}=1 \lambda$.

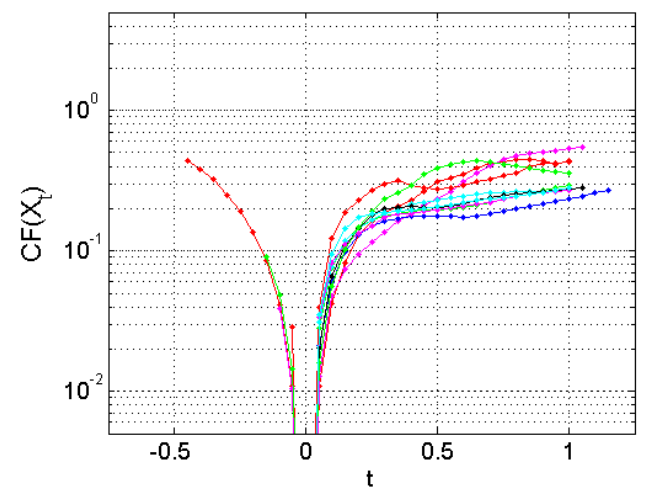

(c) CF (7) evaluation, OUT size, $D_{\text {OUT }}=2 \lambda$.

Fig. 5. Evaluation of cost function (7) for a dielectric body with $\varepsilon_{r}=3$ and $\sigma=0.01 \mathrm{~S} / \mathrm{m}$. Results for 10 random candidates are shown.

minimum.

- The same random seeds are used in all the compared examples. In other words, the $q-t h$ initial population set is the same for all the examples.

- Maximum number of Evolutionary Algorithm (DE, PSO) iterations: 100.

- Evolutionary Algorithm bounding: mirroring. A particle will bounce off when hitting the investigation domain.

For measurements, the PEC OUT profile corresponds to the metallic cylinder described in [24], and the dielectric object is the off-centered bar described in [24]. PSO parameters: i) 


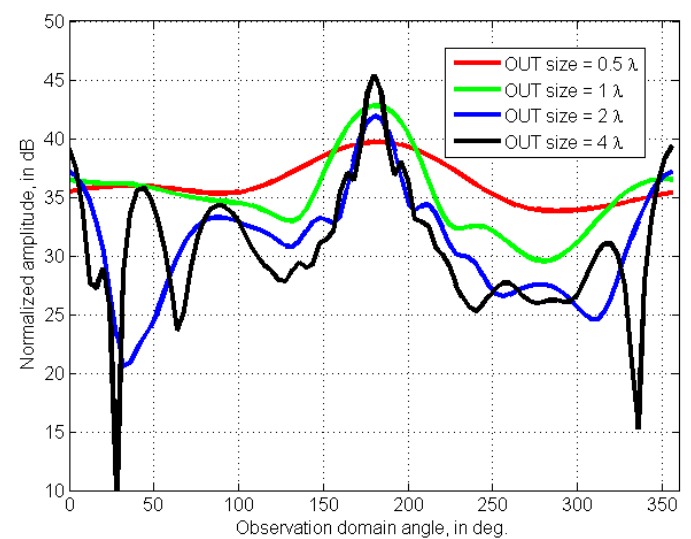

(a) PEC. Scattered field amplitude (in dB).



(b) Dielectric. Scattered field amplitude (in dB).

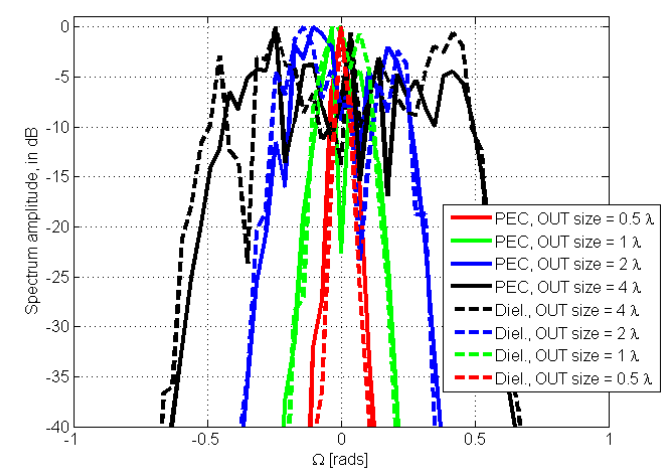

(c) Spectrum of the scattered field (normalized, in $\mathrm{dB}$ ) vs. spatial bandwidth (in rads).

Fig. 6. Comparison between the scattered fields radiated by a PEC and dielectric body having the same geometry. Dashed line: dielectric $\left(\varepsilon_{r}=3\right.$ and $\sigma=0.01 \mathrm{~S} / \mathrm{m})$. Solid line: PEC. correction factor $=2$, ii) inertial weight $=1$. DE parameters: i) crossover probability $=0.8$, ii) scaling factor $=0.6$.

Due to the stochastic nature of PSO and DE, it can be expected that some of the $Q$ initial population sets are not able to converge to the global minimum. For this purpose, the best candidate of all the $Q=100$ initial population sets, together with the average of the $2 / 3(66 \%)$ best candidates are plotted. In the case of the reconstruction of the constitutive parameters, relative permittivity $\varepsilon_{r, r e c}$, and conductivity $\sigma_{r e c}$, results for the $66 \%$ best candidates are averaged.

Concerning calculation time, for every population candidate and for the problem size specified above, cost function evaluation takes around $120 \mathrm{~ms}$ for PEC formulation, and 650 ms for dielectric formulation, in a laptop with 4 GB of RAM and Intel ${ }^{\circledR}$ core i5-3210M at $2.5 \mathrm{GHz}$. Finally, in the case of simulation-based examples, no noise has been added aiming to test if DE or PSO are able to reach the global minimum.

\section{A. PEC}

In the case of the simulation-based example, the amplitude information collected in two circumferences (circular acquisition) of radii $R_{o b s, 1}=15 \mathrm{~cm}$, and $R_{o b s, 2}=30 \mathrm{~cm}$ has been considered. The use of two circumferences is devoted to increase the available amount of information and partially compensate the absence of phase information. Reconstruction results are depicted in Fig. 7 (a), where it can be noticed that both PSO and DE are able to recover the true profile of the $2 \lambda$-size OUT ( $\mathrm{f}=8 \mathrm{GHz}$ ). It can be concluded that, using a large set of initial populations, $Q=100$, it is possible to find a random realization of initial candidates able to converge to the true OUT profile. Fig. 7 (b) represents the cost function convergence as a function of the number of iterations for PSO and DE, respectively. PSO performs slightly better than DE: while best and worse candidates have similar cost function values, the average has smaller value in the case of PSO.

Aiming to provide a reference result, the same example has been tested using the cost function (1). 8 (a) shows the recovered OUT profile for DE and PSO, respectively, using the scattered field information at $R_{o b s, 1}=15 \mathrm{~cm}$ : amplitude and phase information is available, so the improvement with respect to the phaseless case (Fig. 7 (a)) is observed. Again, PSO performs slightly better than DE, as observed as well in Fig. 7 (b) (convergence).

\section{B. Dielectric}

Next, the capability to recover the profile and constitutive parameters of a dielectric object from phaseless data is evaluated. The OUT has the same geometry as the one in Section V-A, electric size of $2 \lambda(\mathrm{f}=8 \mathrm{GHz})$, and constitutive parameters $\varepsilon_{r}=3$ and $\sigma=0.01 \mathrm{~S} / \mathrm{m}$. Two acquisition circumferences of radii $R_{o b s, 1}=15 \mathrm{~cm}$, and $R_{o b s, 2}=30 \mathrm{~cm}$ are considered to partially compensate the lack of information when the phase of the the scattered field is not available. 


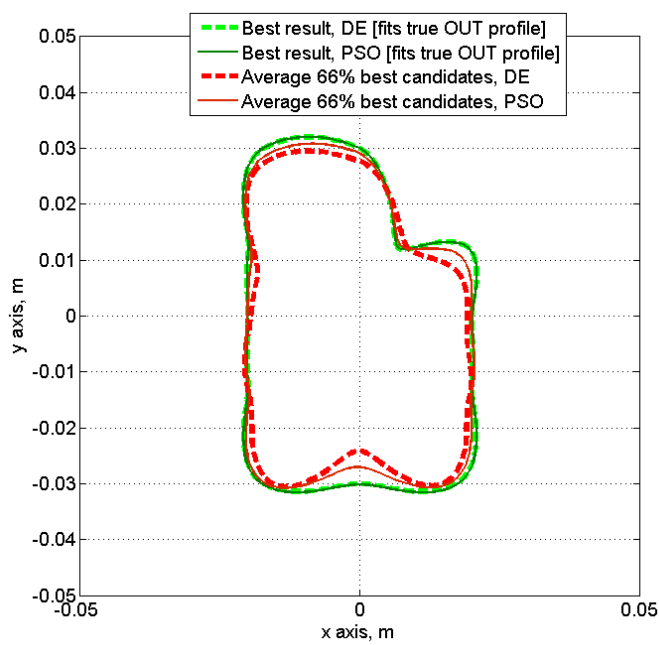

(a) Comparison between DE and PSO (OUT size, $D_{O U T}=2 \lambda$ ).

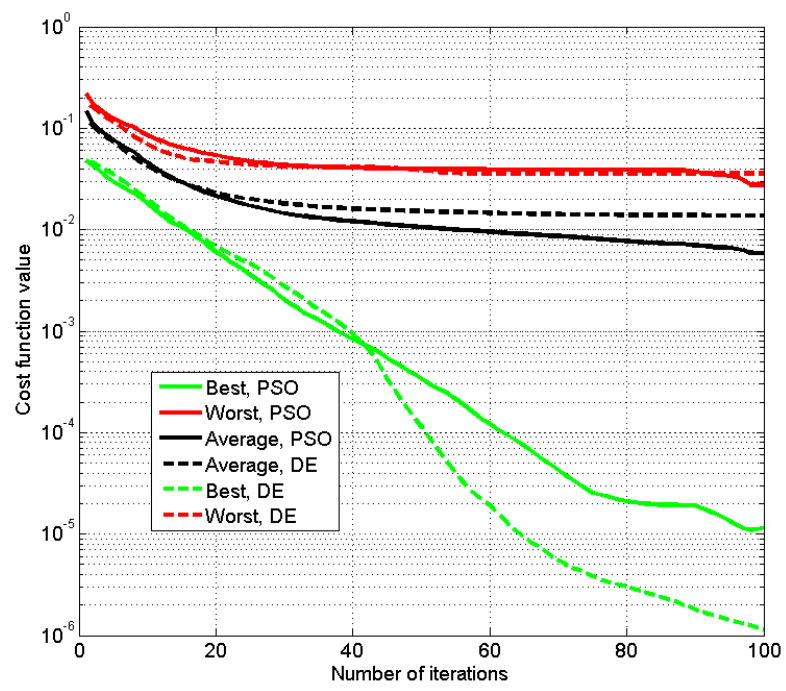

(b) Convergence.

Fig. 7. PEC OUT. Simulation-based example. Profile reconstruction results using amplitude-only information.

Results are depicted in Fig. 9 (a) (average profile for the $66 \%$ best candidates, solid red line) showing that the method fails when trying to recover the profile and the constitutive parameters from phaseless data. Even though the best result (solid green line, Fig.9 (a)) fits the true OUT profile, only 1 out of the 70 initial population sets did converge to the correct solution $\left(\varepsilon_{r, r e c}=2.97\right.$ and $\left.\sigma_{r e c}=0.009 \mathrm{~S} / \mathrm{m}\right)$. On average, the recovered permittivity and conductivity of the $66 \%$ best candidates, $\varepsilon_{r, r e c}=4.80$ and $\sigma_{r e c}=0.076 \mathrm{~S} / \mathrm{m}$, are far from the nominal values.

The reason why the phaseless reconstruction method works for a PEC but not for a dielectric object can be found by analyzing the cost function behavior plotted in Fig. 5. For a dielectric OUT, the cost function (7) has worse behavior for an OUT size of $2 \lambda$ (Fig. 5 (c), with several local minima) than for $1 \lambda$ (Fig. 5 (b), monotonic decrease). Furthermore, the number of incidences was increased from $K=4$ to $K=9$,

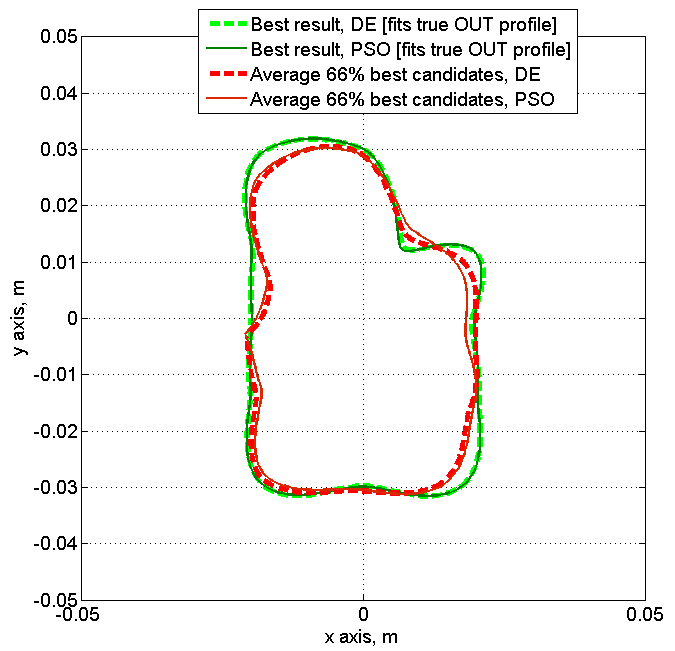

(a) Comparison between DE and PSO (OUT size, $D_{O U T}=2 \lambda$ ).



(b) Convergence.

Fig. 8. PEC OUT. Simulation-based example. Profile reconstruction results using amplitude and phase information.

without noticing an improvement in the reconstruction results.

If the scattered field amplitude information for $1 \lambda$ ( $\mathrm{f}=4$ $\mathrm{GHz}$ ) is considered instead (Fig. 6 (b), green line), it can be expected that the global optimization technique would be able to recover the true OUT profile and constitutive parameters for more than 1 out of 70 initial population sets. Results depicted in Fig. 9 (a) (average profile for the 66\% best candidates, dashed red line) confirm the expected improvement. Now the average conductivity and permittivity values are $\varepsilon_{r, r e c}=3.00$ and $\sigma_{r e c}=0.009 \mathrm{~S} / \mathrm{m}$, in agreement with the nominal OUT values. Notice also the improvement in the cost function convergence when considering an OUT size of $1 \lambda$ with respect to the case of $2 \lambda$, Fig. 9 (b).

PSO and DE have also been benchmarked for this example, with profile reconstruction results and convergence rates similar to the ones for the PEC OUT. Thus, as PSO performs again better than DE, the former global optimization method will be 


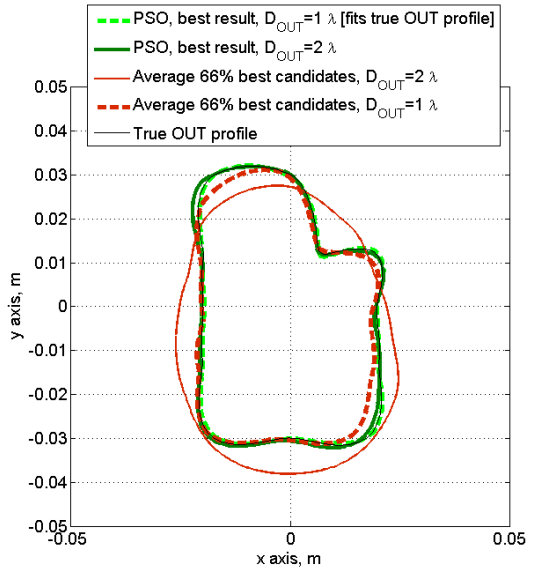

(a) Profile reconstruction results.

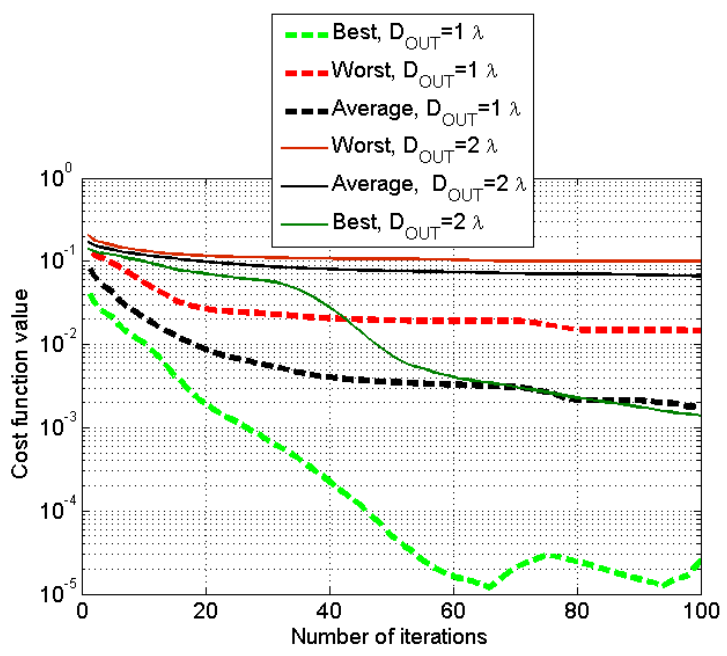

(b) Convergence.

Fig. 9. Dielectric OUT. Simulation-based example.

considered for the rest of reconstruction examples presented in Section VI.

In addition to the two presented examples, the influence of other parameters such as the number of PSO parameters to be optimized -unknowns, $N_{X}$ - and the PSO population size, $P$, has been studied. Reconstruction results, namely mean and maximum profile reconstruction error, and conductivity and permittivity estimation errors, are summarized in Table III. For the analysis presented in Table III, the true OUT profile has been created with the same number of spline control points used later in the reconstruction algorithm; e.g. if the OUT is modeled with 12 spline control points, PSO candidates will have 12 control points as well.

From Table III results it can be noticed that doubling the population size, $P$, has not a significant impact in the reconstruction error. In the case of electrically small $(0.5$ $\lambda)$ or electrically large $(2 \lambda)$ OUTs, reconstruction errors are significantly larger than for $1 \lambda$ size OUT. As justified

\begin{tabular}{|c|c|c|c|c|c|c|}
\hline$D_{\text {OUT }}$ & $N_{X}$ & $P$ & $\epsilon_{\max }(\%)$ & $\epsilon_{\text {mean }}(\%)$ & $\epsilon_{\sigma}(\%)$ & $\epsilon_{\varepsilon, \text { rel }}(\%)$ \\
\hline \multirow{4}{*}{$0.5 \lambda$} & \multirow{2}{*}{16} & 50 & 55 & 19 & 690 & 41 \\
\hline & & 100 & 53 & 18 & 600 & 39 \\
\hline & \multirow{2}{*}{10} & 50 & 36 & 15 & 530 & 34 \\
\hline & & 100 & 38 & 13 & 500 & 32 \\
\hline \multirow{4}{*}{$1 \lambda$} & \multirow{2}{*}{16} & $50^{\dagger}$ & 11 & 5 & 10 & $<1$ \\
\hline & & 100 & 13 & 6 & 11 & $<1$ \\
\hline & \multirow{2}{*}{10} & 50 & 15 & 6 & 41 & 5 \\
\hline & & 100 & 12 & 6 & 40 & 4 \\
\hline \multirow{4}{*}{$2 \lambda$} & \multirow{2}{*}{16} & $50^{\dagger}$ & 35 & 17 & 660 & 60 \\
\hline & & 100 & 42 & 15 & 620 & 57 \\
\hline & \multirow{2}{*}{10} & 50 & 33 & 16 & 200 & 23 \\
\hline & & 100 & 27 & 15 & 190 & 20 \\
\hline
\end{tabular}

Error is defined as: $\epsilon=\mid$ Recovered - Reference $\mid /$ Reference.

$\epsilon_{\max }$ : maximum error between the true and reconstructed OUT profile.

$\epsilon_{\text {mean }}$ : mean error between the true and reconstructed OUT profile. $\epsilon_{\sigma}$ :

conductivity reconstruction error. $\epsilon_{\varepsilon, r e l}$ : relative permittivity

reconstruction error. Note ${ }^{\dagger}$ : full example presented in Section V-B.

TABLE III

RECONSTRUCTION ERROR FOR DIFFERENT DIELECTRIC OUT SIZES $\left(D_{O U T}\right)$ AS A FUNCTION OF THE NUMBER OF UNKNOWNS $\left(N_{X}\right)$ AND PSO POPULATION SIZE $(P)$.

in Section IV, this is directly related to the impact of the OUT electric size on the scattered field amplitude aspect angle variation, which eventually influences the cost function behavior.

\section{Comparison with existing inverse scattering methods}

Aiming to compare the performance of the proposed monochromatic phaseless inverse scattering with an inversion method that requires amplitude and phase information, the first example of Section IV of [19] has been selected. The OUT is a homogeneous dielectric cylinder centered at $\left(x_{c}, y_{c}\right)=$ $(-0.055,0.055) \lambda$, with $D_{O U T}=0.66 \lambda$ diameter. The contrast function has a value of 1 , so the relative permittivity is $\varepsilon_{r}=2$. The field scattered by the OUT is depicted in Fig. 2(b) of [19], observing a pattern similar to the one shown in Fig. 6 (b) due to the 1- $\lambda$ OUT size. The scattered field is acquired at one single circumferential domain of $R_{o b s}=0.83 \lambda$. Thus, for this example, phaseless reconstruction definitely implies a loss of information with respect to Section V-A and Section V-B examples, where two acquisition surfaces were considered for phaseless reconstruction.

In [19] a Genetic Algorithm (GA) is proposed for minimizing a cost function that essentially relates the same parameters as cost function (1). Pixel-based discretization is considered, with 9x9 unknown parameters. For comparison purposes, the same population size, $P=80$, is considered. The rest of the inverse problem parameters is summarized in Table II.

First, amplitude and phase information has been considered aiming to test the PSO capability to find the global minimum. In the $Q=70$ evaluated cases, PSO was able to converge 
to the global minimum, with a profile reconstruction error $<1.5 \%$, and a recovered permittivity and conductivity of $\varepsilon_{r, r e c}=2.0$ and $\sigma_{r e c}=8.8 \times 10^{-6} \mathrm{~S} / \mathrm{m}$. Next, phaseless capabilities have been tested by minimizing cost function (7). Reconstruction results are depicted in Fig. 10 (a), with an average reconstruction error for the $66 \%$ best candidates of $7 \%$. The average permittivity and conductivity values are $\varepsilon_{r, r e c}=2.26$ and $\sigma_{r e c}=0.003 \mathrm{~S} / \mathrm{m}$, respectively. Aiming to improve convergence, the number of spline control points was decreased from 12 to 6 , thus reducing the number of unknowns from $N_{X}=16$ to $N_{X}=10$. As depicted in Fig. 10 (b), it can be observed that convergence improved, with an average reconstruction error for the $66 \%$ best candidates of $2 \%, \varepsilon_{r, r e c}=2.16$ and $\sigma_{r e c}=0.001 \mathrm{~S} / \mathrm{m}$. For both $N_{X}=16$ and $N_{X}=10$, the recovered permittivity and OUT profile are in agreement with the results presented in Fig. 3 of [19], with the advantage that the results of Fig. 10 have been recovered from phaseless measurements.

From the results of this subsection it can be concluded that the number of unknowns also influences the convergence of the global optimization technique. OUTs with slow-varying geometry with respect to the aspect angle can be parametrized with fewer spline control points. However, this information is not known a-priori, as the scattered field spatial bandwidth depends mainly on the OUT electric size, rather than the OUT shape.

\section{Validation with Measurements}

\section{A. Fresnel Institute Dataset}

An example from the Fresnel Institute dataset [24] has been selected to evaluate profile and constitutive parameters reconstruction capability using measurements. In this case, the OUT is a dielectric cylinder, centered at $\left(x_{c}, y_{c}\right)=(0,0.03)$ $\mathrm{m}$, with $D_{\text {OUT }}=0.03 \mathrm{~cm}$ diameter. The relative permittivity is $\varepsilon_{r}=3 \pm 0.3$. Results for OUT electric sizes of $0.4 \lambda, 0.8$ $\lambda$, and $1.2 \lambda$ (corresponding to $f=4 \mathrm{GHz}, 8 \mathrm{GHz}$, and 12 $\mathrm{GHz}$ ), have have been studied. In this subsection, in addition to the best and average results for all the initial population sets, best candidates results for each initial population set are also plotted (dashed pink line). Initially, $K=4$ incidences were considered, but the OUT circular profile was not accurately reconstructed. In consequence, it was decided to test the inversion method using $K=9$ incident directions.

Phaseless reconstruction results for $0.8 \lambda$ are depicted in Fig. 11 (b): the results for each individual population set (dashed pink line) exhibit some dispersion with respect to the true solution, although the average of the $66 \%$ best candidates (light blue line) as well as the best candidate (green line) fit the true OUT position and shape. Concerning the recovery of the constitutive parameters, estimated permittivity, $\varepsilon_{r, \text { rec }}=2.98$, is within the tolerance limits $\left(\varepsilon_{r}=3 \pm 0.3\right)$ [20] .

However, for $0.4 \lambda$, neither the location (shifted $0.75 \mathrm{~cm}$ towards $+\mathrm{y})$ nor the constitutive parameters $\left(\varepsilon_{r, r e c}=3.98\right)$ are properly recovered, as shown in Fig. 11 (a). At this frequency, the OUT scattered field amplitude has little spatial variation, so different candidates create similar, slow-varying scattered field

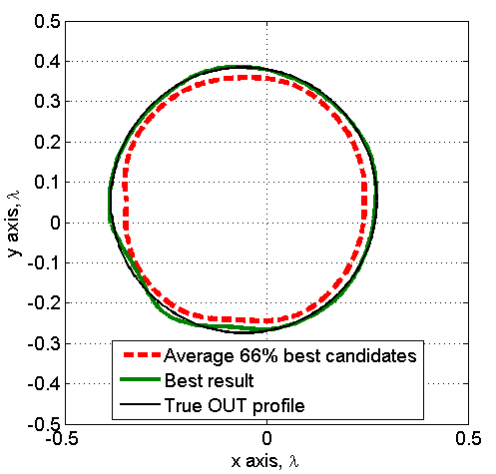

(a) $N_{X}=16$ unknowns, OUT size, $D_{O U T}=0.66 \lambda$. $\varepsilon_{r, r e c}=2.26$ and $\sigma_{\text {rec }}=0.003 \mathrm{~S} / \mathrm{m}$.

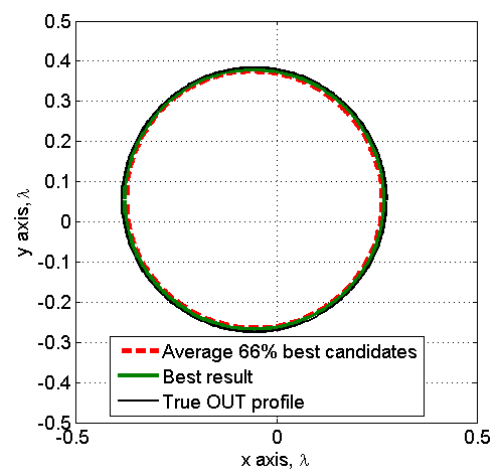

(b) $N_{X}=10$ unknowns, OUT size, $D_{\text {OUT }}=0.66 \lambda$. $\varepsilon_{r, \text { rec }}=2.16$ and $\sigma_{r e c}=0.001 \mathrm{~S} / \mathrm{m}$.

Fig. 10. Dielectric OUT (Ref. [19], Section IV, first example). Profile and constitutive parameters reconstruction from simulated data.

amplitude (as explained in Section IV). Finally, reconstruction using measurements for an OUT size of $1.2 \lambda$, where the OUT scattered field amplitude exhibits faster spatial variation, has been done. Results depicted in Fig. 11 (c) confirm that, not only the constitutive parameters, $\varepsilon_{r, r e c}=3.04$, but also the averaged profile are accurately recovered. However, due to the faster spatial variation of the scattered field amplitude, some candidates are not able to reach the global minimum.

\section{B. Implemented Measurement Setup}

Finally, reconstruction capabilities using the measurements collected with the setup presented in Section II are presented. First, reconstruction results for the metallic bar $\left(D_{O U T}=2.2\right.$ $\mathrm{cm}(0.7 \lambda$ at the working frequency of $\mathrm{f}=9.4 \mathrm{GHz})$ are plotted in Fig. 12 for each initial population set (dashed pink line), comparing it with the true profile of the metallic bar (solid black line). Different initial population sets did not converge to the same profile, although, on average, the metallic bar profile (dashed blue line) is recovered. For the $66 \%$ best candidates, the maximum profile reconstruction error is $\epsilon_{\max }=12 \%$, and the average error is $\epsilon_{\text {mean }}=9 \%$. It must be remarked that this object has been considered primarily for calibration and initial testing of the phaseless measurement setup. 


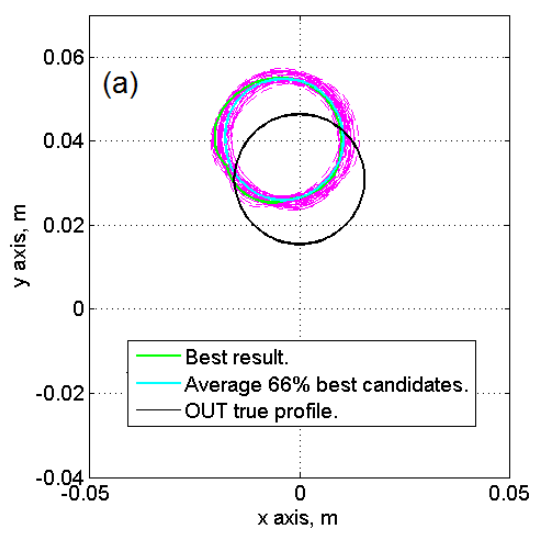

(a) $K=9$ incidences, OUT size, $D_{O U T}=0.4 \lambda$. $\varepsilon_{r, \text { rec }}=3.98$ and $\sigma_{r e c}=0.104 \mathrm{~S} / \mathrm{m}$.

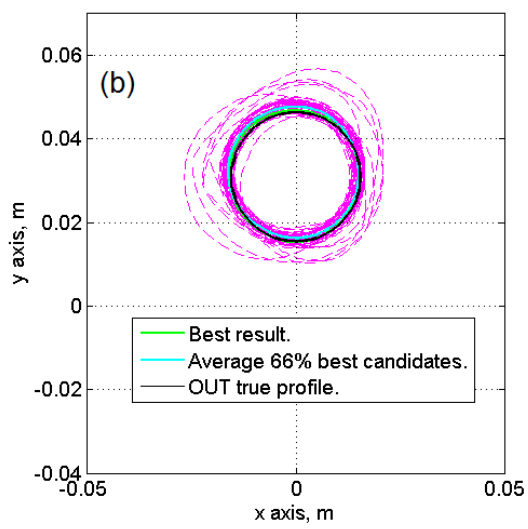

(b) $K=9$ incidences, OUT size, $D_{O U T}=0.8 \lambda$. $\varepsilon_{r, \text { rec }}=2.98$ and $\sigma_{r e c}=0.025 \mathrm{~S} / \mathrm{m}$.

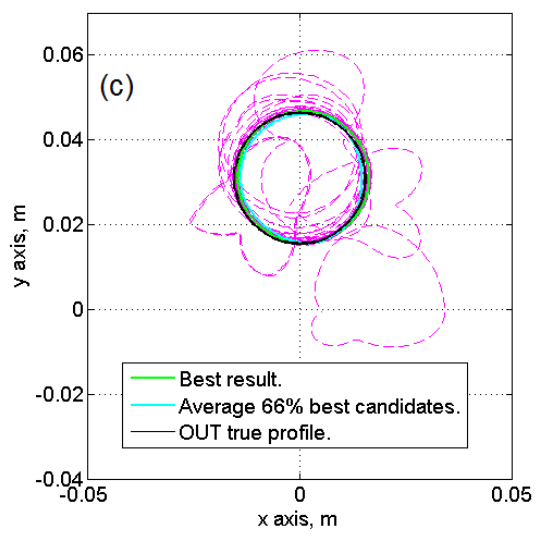

(c) $K=9$ incidences, OUT size, $D_{O U T}=1.2 \lambda$.

$$
\varepsilon_{r, r e c}=3.04 \text { and } \sigma_{r e c}=0.019 \mathrm{~S} / \mathrm{m} \text {. }
$$

Fig. 11. Dielectric OUT. Profile and constitutive parameters reconstruction from measurements. Best candidate results (pink dashed line) for each initial population set.
The capability of the measurement setup for profile and constitutive parameters reconstruction has been validated by means of two dielectric objects: a wood stick (see Fig. 2 (c)), and a wax candle with square cross section (Fig. 2 (b)). The recovered profile of the wood stick $\left(D_{O U T}=1.5 \lambda\right)$ for each initial population set is depicted in Fig. 13 (dashed pink lines), and compared to the true profile (solid black line). As in previous examples, the $66 \%$ best candidates are considered for quantitative analysis. The fact of parameterizing a rectangular cross-section with splines yields a larger maximum reconstruction error, $\epsilon_{\max }=33 \%$, although the average reconstruction error is significantly smaller $\epsilon_{\text {mean }}=9 \%$. Concerning the constitutive parameters, the averaged recovered permittivity and conductivity are $\varepsilon_{r, r e c}=2.78$ and $\sigma_{r e c}=0.33 \mathrm{~S} / \mathrm{m}$, respectively, close to the range $\varepsilon_{r}=2.5 \pm 0.1$ and $\sigma=$ $0.3 \pm 0.03 \mathrm{~S} / \mathrm{m}^{1}$ given in Fig. 7 of [34] (gray line, plywood). It must be taken into account that materials of the same category measured by different researchers may not have the same composition, hence differences in the results might indeed be expected.

Finally, results for the wax candle $\left(D_{O U T}=3.5 \lambda\right)$ are depicted in Fig. 14. It can be noticed the large dispersion between the results for each initial population set (dashed pink lines), due to the larger OUT electric size (the largest among all the examples of this manuscript, see Table IV). Results for $33 \%$ and $66 \%$ best candidates are also depicted, the latter having a significant deviation from the nominal OUT profile. For the $66 \%$ best candidates, permittivity and conductivity are $\varepsilon_{r, r e c}=2.02$ and $\sigma_{r e c}=0.03 \mathrm{~S} / \mathrm{m}$, and for the $33 \%$ best candidates, $\varepsilon_{r, r e c}=2.17$ and $\sigma_{r e c}=0.03 \mathrm{~S} / \mathrm{m}$, respectively. Recovered permittivity values are in agreement with the expected for wax (paraffin) $\left(\varepsilon_{r}=2.3 \pm 0.2\right)$ [35] and Table 6.1 of [36].

Apart from the square cross-section wax candle, another with cylindrical cross-section $\left(D_{O U T}=6 \mathrm{~cm}\left(\begin{array}{ll}1.9 & \lambda\end{array}\right)\right)$ has been measured as well. Reconstruction errors for the $66 \%$ best candidates are summarized in Table IV, where it can be noticed the smaller profile reconstruction error with respect to the square wax candle, not only because the smoother profile, but also because of the smaller electric size. Recovered constitutive parameters are $\varepsilon_{r, r e c}=2.5$ and $\sigma_{r e c}=0.005 \mathrm{~S} / \mathrm{m}$, again in agreement with the expected for wax.

\section{CONCLUSIONS}

A method and low-cost measurement setup for profile and constitutive parameters reconstruction from monochromatic phaseless measurements has been presented. The method is based on the minimization of a cost function relating the measured received power or scattered field amplitude with the one calculated using an integral equation model of the

\footnotetext{
${ }^{1}$ From the loss tangent, $\delta$, the conductivity is given by: $\sigma=$ $2(2 \pi f) \varepsilon_{r} \varepsilon_{0} \delta=2(2 \pi 9.4 G H z)[2.5 \pm 0.1][0.115 \pm 0.005]=[0.3 \pm$ $0.03] \mathrm{S} / \mathrm{m}$
} 


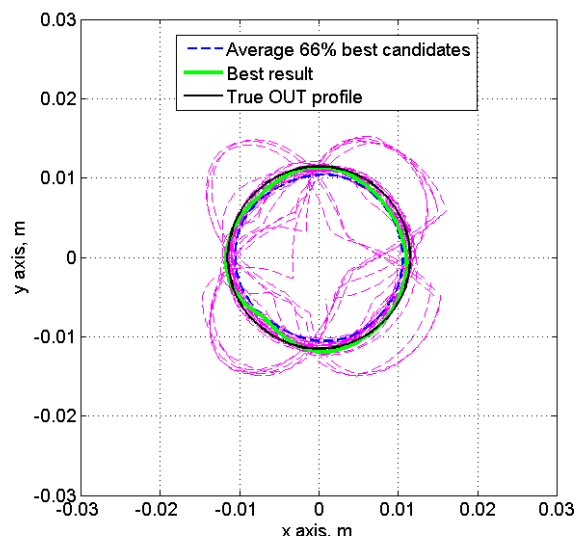

Fig. 12. Metallic bar. Recovered profile.

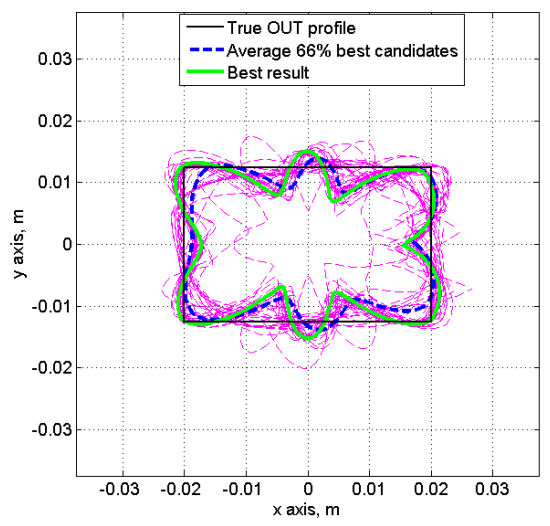

Fig. 13. Wood stick. Recovered profile.

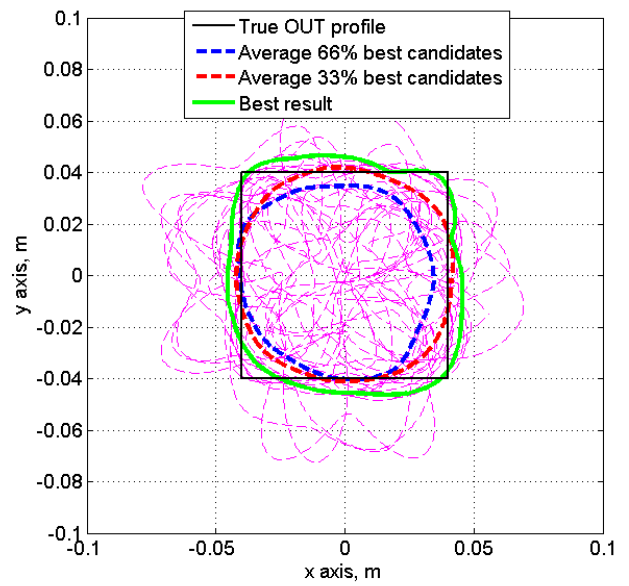

Fig. 14. Wax candle. Recovered profile. parametrized OUT. Two stochastic global optimization techniques, DE and PSO, have been evaluated, concluding that PSO performs, on average, slightly better than DE.

From the results presented in this contribution, and summarized in Table IV, the proposed reconstruction method is capable of recovering the OUT profile and constitutive parameters for scatterers having around one wavelength electric size. For this size, the scattered field amplitude has significant amplitude fluctuation (that is, enough information about the OUT), but not too much spatial variation so the global optimization method gets stuck in a local minimum. This dependence with the electric size of the OUT is also correlated with the behavior of the cost function being minimized.

Measurement results confirm the feasibility of the developed technique, conceived to be used with single-frequency measurements collected using power detectors, thus enabling the use of such a simple, low-cost measurement setup, as the one presented in this paper.

\section{REFERENCES}

[1] Nikolova, N., "Microwave imaging for breast cancer," IEEE Microwave Magazine, Vol. 7, No. 12, pp. 78-94, 2011.

[2] Henriksson, T., Joachimowicz, N., Conessa, C., \& Bolomey, J. C., "Quantitative microwave imaging for breast cancer detection using a planar $2.45 \mathrm{GHz}$ system," IEEE Transactions on Instrumentation and Measurement, Vol. 59, No. 10, pp. 2691-2699, 2010.

[3] Gonzalez-Valdes, B., Alvarez, Y., Gutierrez-Meana, J., Rappaport, C., Las Heras, F., Pino, A. G., \& Martinez-Lorenzo, J. A., "On-themove millimeter wave imaging system using multiple transmitters and receivers," 2015 9th European Conf. on Antennas and Propagation $(E u C A P)$, Lisbon, Portugal, April 2015. pp. 1-4.

[4] Sheen, D. M., McMakin, D. L., Hall, T. E., \& Severtsen, R. H. "Active millimeter-wave standoff and portal imaging techniques for personnel screening," . 2009 IEEE Technologies for Homeland Security Conference (HST'09). May 2009, pp. 440-447.

[5] Ahmed, S. S., Schiessl, A., Gumbmann, F., Tiebout, M., Methfessel, S., \& Schmidt, L., "Advanced microwave imaging," IEEE Microwave Magazine, Vol. 13, No. 6, pp. 26-43, 2012.

[6] Soldovieri, F., Solimene, R., \& Ahmad, F., "Sparse tomographic inverse scattering approach for through-the-wall radar imaging," IEEE Transactions on Instrumentation and Measurement, Vol. 61, No. 12, pp. 33403350, 2012.

[7] Benedetti, M., Donelli, M., Martini, A., Pastorino, M., Rosani, A., \& Massa, A., "An innovative microwave-imaging technique for nondestructive evaluation: applications to civil structures monitoring and biological bodies inspection," IEEE Transactions on Instrumentation and Measurement, Vol. 55, No. 6, pp. 1878-1884, 2006.

[8] Salvadè, A., Pastorino, M., Monleone, R., Bozza, G., \& Randazzo, A., "A new microwave axial tomograph for the inspection of dielectric materials," IEEE Transactions on Instrumentation and Measurement, Vol. 58, No. 7, pp. 2072-2079, 2009.

[9] Laviada, J., Arboleya-Arboleya, A., Alvarez-Lopez, Y., GarciaGonzalez, C., \& Las-Heras, F., "Phaseless Synthetic Aperture Radar With Efficient Sampling for Broadband Near-Field Imaging: Theory and Validation," IEEE Transactions on Antennas and Propagation, Vol.63, No .2, pp.573-584, Feb. 2015.

[10] Crocco, L., D'Urso, M., \& Isernia, T., "Faithful non-linear imaging from only-amplitude measurements of incident and total fields," Optics Express, Vol. 15, No. 7, pp. 3804-3815, 2007.

[11] Franceschini, G., Donelli, M., Franceschini, D., Benedetti, M., Rocca, P., \& Massa, A., "Microwave imaging from amplitude-only data-advantages and open problems of a two-step multi-resolution strategy," Progress In Electromagnetics Research PIER, Vol. 83, pp. 397-412, 2008.

[12] Hislop, G., Li, L., \& Hellicar, A., "Phase retrieval for millimeterand submillimeter-wave imaging", IEEE Transactions on Antennas and Propagation, Vol. 57, No. 1, pp. 286-290, 2009.

[13] Crocco, L., D’Urso, M., \& Isernia, T., "Inverse scattering from phaseless measurements of the total field on a closed curve," Journal of the Optical Society of America American, Vol. 21, No. 4, pp. 622-631, April 2004. 


\begin{tabular}{|c|c|c|c|c|c|c|c|c|}
\hline & $D_{O U T}$ & Method & $N_{X}$ & $\epsilon_{\max }(\%)$ & $\epsilon_{\text {mean }}(\%)$ & $\epsilon_{\sigma}(\%)$ & $\epsilon_{\varepsilon, r e l}(\%)$ & Section \\
\hline \multirow{3}{*}{ PEC } & $0.7 \lambda$ & PSO & 8 & 12 & 9 & N/A & N/A & VI-B \\
\hline & \multirow{2}{*}{$2 \lambda$} & PSO & 14 & 10 & 3 & N/A & N/A & $\mathrm{V}-\mathrm{A}$ \\
\hline & & $\mathrm{DE}$ & 14 & 11 & 3 & N/A & N/A & $\mathrm{V}-\mathrm{A}$ \\
\hline \multirow{10}{*}{ Diel. } & $0.4 \lambda$ & PSO & 10 & 73 & 39 & Unkn. & $\begin{array}{c}33^{\dagger} \\
\text { Ref. val. tol.: } 20 \text { [20] }\end{array}$ & VI-A \\
\hline & $0.66 \lambda$ & PSO & 10 & 5 & 3 & Inf. & 8 & V-C \\
\hline & $0.66 \lambda$ & PSO & 16 & 12 & 7 & Inf. & 13 & $\mathrm{~V}-\mathrm{C}$ \\
\hline & $0.8 \lambda$ & PSO & 10 & 9 & 5 & Unkn. & $\begin{array}{c}<1^{\dagger} \\
\text { Ref. val. tol.: } 20[20]\end{array}$ & VI-A \\
\hline & \multirow{2}{*}{$1 \lambda$} & PSO & 16 & 11 & 5 & 10 & $<1$ & V-B \\
\hline & & $\mathrm{DE}$ & 16 & 16 & 7 & 10 & $<1$ & V-B \\
\hline & $1.2 \lambda$ & PSO & 10 & 10 & 5 & Unkn. & $\begin{array}{c}1^{\dagger} \\
\text { Ref. val. tol.: } 20 \text { [20] }\end{array}$ & VI-A \\
\hline & $1.5 \lambda$ & PSO & 16 & 33 & 9 & $\begin{array}{c}10 \\
\text { Ref. val. tol.: } 20 \text { [34] }\end{array}$ & $\begin{array}{c}11^{\dagger} \\
\text { Ref. val. tol.: } 8 \text { [34] }\end{array}$ & VI-B \\
\hline & $1.9 \lambda$ & PSO & 10 & 16 & 11 & Unkn. & $\begin{array}{c}9^{\dagger} \\
\text { Ref. val. tol.: } 17 \text { [35],[36] }\end{array}$ & VI-B \\
\hline & $3.5 \lambda$ & PSO & 16 & 38 & 16 & Unkn. & $\begin{array}{c}12^{\dagger} \\
\text { Ref. val. tol.: } 17 \text { [35],[36] }\end{array}$ & VI-B \\
\hline
\end{tabular}

N/A: not applicable. Unkn.: the reference value is not known. Inf.: true OUT conductivity equal to $0 \mathrm{~S} / \mathrm{m}$. Ref. val. tol.: reference value tolerance, in $\%$, defined as Tolerance $=$ Range $/$ Mean value (e.g. for $\varepsilon_{r}=2.3 \pm 0.2$ tolerance is $100 \mathrm{x} 0.4 / 2.3=17$ ). Note $e^{\dagger}$ : error with respect to the mean value of the reference range.

TABLE IV

SUMMARY OF THE RECONSTRUCTION RESULTS ERRORS CONSIDERING THE AVERAGE OF 66\% BEST CANDIDATES.

[14] Costanzo, S., Di Massa, G., Pastorino, M., \& Randazzo, A., "Hybrid Microwave Approach for Phaseless Imaging of Dielectric Targets," IEEE Geoscience and Remote Sensing Letters, Vol. 12, No. 4, pp. 851-854, 2015.

[15] Álvarez, Y., García, C., \& Las-Heras, F., "Geometry reconstruction from amplitude-only scattered field data," In Proc. of the 5th European Conference on Antennas and Propagation (EuCAP'11), Rome (Italy), 9-15 April 2011, pp. 1-5.

[16] Rocca, P., Oliveri, G., \& Massa, A, "Differential evolution as applied to electromagnetics," IEEE Antennas and Propagation Magazine, Vol. 53, No. 1, pp. 38-49, 2011.

[17] Rekanos, I. T., "Shape reconstruction of a perfectly conducting scatterer using differential evolution and particle swarm optimization," IEEE Transactions on Geoscience and Remote Sensing, Vol. 46, No. 7, pp. 1967-1974, 2008.

[18] Garcia-Fernandez, M., Garcia, C., Alvarez, Y, \& Las-Heras, F., "Influence of contour smoothness and electric size on the profile reconstruction of metallic objects using hybrid optimization," in EUROCON 2015, IEEE International Conference on Computer as a Tool, Salamanca (Spain), 8-11 September 2015, pp.1-6.

[19] Pastorino, M., Massa, A., \& Caorsi, S., "A microwave inverse scattering technique for image reconstruction based on a genetic algorithm, "IEEE Transactions on Instrumentation and Measurement, Vol. 49, No. 3, pp. 573-578, 2000.

[20] Marklein, R., Balasubramanian, K., Qing, A., \& Langenberg, K. J. "Linear and nonlinear iterative scalar inversion of multi-frequency multibistatic experimental electromagnetic scattering data," Inverse Problems, vol. 17, no. 6, pp. 1597-1610, 2001.

[21] Chiu, C. C., \& Liu, P. T., "Image reconstruction of a perfectly conducting cylinder by the genetic algorithm," IEE Proceedings - Microwave, Antennas and Propagation, Vol. 143, No. 3, pp. 249-253, 1996.

[22] Pastorino, M., Caorsi, S., \& Massa, A., "A global optimization technique for microwave nondestructive evaluation," IEEE Transactions on Instrumentation and Measurement, Vol. 51, No. 4, pp. 666-673, 2002.

[23] Pastorino, M., "Short-range microwave inverse scattering techniques for image reconstruction and applications," IEEE Transactions on Instrumentation and Measurement, Vol. 47, No. 6, pp. 1419-1427, 1998.

[24] Belkebir, K., \& Saillard, M. "Special section: Testing inversion algo- rithms against experimental data," Inverse Problems, vol. 17, no. 6, pp. $1565-1571,2001$

[25] LD Didactic. CassyLab antenna measurement setup. Available at: http://www.ld-didactic.de/documents/en-US/GA/GA/7/737/737405e.pdf (accessed 27-06-2016).

[26] LD Didactic. Small (73720) and Large (73721) Horn Antenna. Available at: http://www.ld-didactic.de/documents/enUS/GA/GA/7/737/73720e.pdf (accessed 27-06-2016).

[27] Leybold Coax Detector 737 03: http://www.leyboldshop.com/technology/electrical-engineering/telecommunicationstechnology/microwave-technology/wave-guide-technology/coaxdetector-73703.html (accessed 27-06-2016).

[28] Balanis, C. A., Advanced Engineering Electromagnetics, John Wiley \& Sons, 1989.

[29] Harrington, R. F., Field Computation by Moment Methods, Wiley-IEEE Press, 1993.

[30] Trinh, D., Accelerated Integral Equation Techniques for solving EM Wave Prppagation and Scattering Problems. Doctoral dissertation, Dublin City University, 2004.

[31] Michalski, K. A, "Electromagnetic imaging of elliptical-cylindrical conductors and tunnels using a differential evolution algorithm," Microwave and Optixal Technology Letters, Vol. 28, No. 3, 2001, pp. 164-169.

[32] Vesterstrøm, J., \& Thomsen, R., "A comparative study of differential evolution, particle swarm optimization, and evolutionary algorithms on numerical benchmark problems," In IEEE Congress on Evolutionary Computation, CEC2004. June 2004. Vol. 2, pp. 1980-1987.

[33] Ebbesen, S., Kiwitz, P. \& Guzzella, L., "A Generic Particle Swarm Optimization Matlab Function," Proceedings of the 2012 American Control Conference, June 27-29, Montreal, Canada, pp. 1514-1524.

[34] Muqaibel, A., Safaai-Jazi, A., Attiya, A. M., \& Riad, S. M., "Ultrawideband through-the-wall propagation," IEE Proceedings-Microwave Antennas and and Propagation, Vol. 152, No. 6, 2005, pp. 581-588.

[35] Banerjee, P., Ghosh, G., \& Biswas, S. K., "A Simple Method to Determine the Dielectric Constant of Small-Sized Medium-Loss Samples at X-Band Frequencies," International Journal of Electromagnetics and Applications, Vol. 1, No. 1, pp. 12-15, 2011.

[36] Brodie, G., Jacob, M. V., \& Farrell, P., Microwave and Radio-Frequency Technologies in Agriculture: An Introduction for Agriculturalists and Engineers. Walter de Gruyter GmbH \& Co KG. 2016. 